\title{
Imbalanced Feshbach-resonant Fermi gases
}

\author{
Leo Radzihovsky \\ Department of Physics, University of Colorado, Boulder, Colorado 80309 \\ E-mail: radzihov@colorado.edu
}

Daniel E. Sheehy

Department of Physics and Astronomy, Louisiana State University, Baton Rouge, Louisiana 70803

E-mail: sheehy@lsu.edu

\begin{abstract}
.
We present an overview of recent developments in species-imbalanced ("polarized") Feshbach-resonant Fermi gases. We summarize the current status of thermodynamics of these systems in terms of a phase diagram as a function of the Feshbach resonance detuning, polarization and temperature. We review instabilities of the swave superfluidity across the BEC-BCS crossover to phase separation, FFLO states, polarized molecular superfluidity and the normal state, driven by the species imbalance. We discuss different models and approximations of this system and compare their predictions to current experiments.
\end{abstract}




\section{Introduction}

\subsection{Background and motivation}

Launched by the laboratory achievement of Bose-Einstein condensation (BEC) in dilute alkali gases [1, 2], atomic physics has undergone nothing short of a revolution. It has transitioned from a few-body science to the study of many-body physics featuring thermodynamic phases and phase transitions. Some of the most exciting experimental developments have included observations of lattices of quantized vortices in rotating BEC gases [3, 4, 5, 6], the Mott insulator to superfluid transition of bosons in an optical lattice [7, 8, 9], the creation of a Fermi sea of cold fermionic atoms [10], and the observation of paired fermionic superfluids that mimic superconductivity in a metal or superfluidity of ${ }^{4} \mathrm{He}$ [11, 12, 13, 14, 15, 16, 17, 18,.

After an early period exploring weakly interacting BEC's and Fermi gases, recently much attention has focused on strong interactions in degenerate atomic gases, which can be realized, for example, by confining the atoms with a periodic optical potential [19], simulating a solid state crystal. A complementary way to explore strong interactions [20, 21, 22] in atomic gases is through the use of Feshbach resonances (FR) [23], abundant in alkali atoms [24, 25, 26], which allow the experimental control of the inter-atomic interactions. This crucial feature of a FR, its tunability, arises through the Zeeman shift (detuning, $\delta$ ) of a diatomic molecular "closed"-channel bound state relative to the "open"-channel atomic continuum. This allows a degree of control (even in real time) over the strength and sign of the open-channel atomic interactions [27, 28, 29, 30], that is unprecedented in other (e.g., solid-state) contexts.

Feshbach resonance tunability has led to laboratory realizations and theoretical proposals for a broad range of quantum many-body phenomena. The most notable experimental realization is of fermion-paired $s$-wave resonant superfluidity, that exhibits the BEC-to-Bardeen-Cooper-Schrieffer (BEC-BCS) crossover between a coordinatespace-paired condensate of dilute diatomic molecules (for large negative detuning) to a Fermi-surface momentum-paired BCS regime of strongly overlapping Cooper pairs (for large positive detuning) [31, 32, 33, 34, 35, 36, 27, 37, 38, 39]. This remarkable controllability of the atomic interactions comes along with other tunable properties, including the background potential (that can be used to confine an atomic gas to one [40] or two dimensions [41], or to impose a periodic optical potential), and the atom numbers of the trapped atomic species.

The focus of this review is an extremely successful set of experiments studying species-imbalanced ("polarized" [42]) mixtures of two hyperfine states of Feshbachresonantly interacting fermionic gases. The two hyperfine states form a pseudo-spin $1 / 2$ system, that is thus closely related to electrons in a metal [43] or high-density quark matter [4], but with the advantage of the tunability of the interactions and trapping potential.

Thus this research effort elucidates the response of interacting fermions to the frustration introduced by species imbalance, that despite attractive interactions prevents 
a complete pairing and drives phase transitions to a variety of interesting quantum ground states and thermodynamic phases. A sufficiently large species imbalance is predicted (and observed) to destroy superfluid order of resonantly interacting fermions even at zero temperature. Understanding the resulting strongly interacting nonsuperfluid ground state is of considerable interest and may shed light on other strongly correlated states lacking broken symmetry such as the "pseudogap" and marginal-Fermi liquid regimes observed in high $\mathrm{T}_{c}$ cuprate superconductors [45, 46], heavy-fermion and Kondo-lattice systems [47, 48], and the phenomenology near quantum critical points [49].

By identifying the species imbalance, $\Delta N$, with the magnetization $M(=\Delta N / V$ with $V$ the system volume) and the two-species chemical potential difference, $\Delta \mu=$ $\mu_{\uparrow}-\mu_{\downarrow} \equiv 2 h$ with an effective Zeeman energy $h$, this research makes a connection to a large body of work on a related condensed-matter system, namely superconductors under an applied magnetic field [43. Thus, imbalanced Feshbach-resonant atomic systems allow a natural extension of superconductivity in a Zeeman field (previously limited to the BCS regime [50, 51]) across the full BEC-BCS crossover. Furthermore, unlike charged solid-state superconductors, where Zeeman and orbital fields are necessarily simultaneously induced by the applied magnetic field, atomic systems are advantageous as they permit independent control of these two effects, with the former created by species chemical potential difference, and the latter induced by the rotation of the atomic cloud [52, 53, 54].

Experiments on polarized Fermi gases have been led by the MIT and Rice groups [55, 56, 57, 58], that early on observed imbalance-induced phase-separation and explored theoretical predictions (such as the phase diagram [59]) for the BEC-BCS crossover at a finite species imbalance [60, 61, 62, 63, 64, 65, 66, 67]. In such experiments, the system is typically prepared with all the atoms in one state. By applying a radiofrequency $(\mathrm{RF})$ pulse [55, 56], a controllable number of atoms can be transferred to the other state, creating an imbalanced Fermi gas. The error on the resulting imbalance (measured at the end of the experiment) is typically a few percent, allowing an accurate study of the phases of strongly interacting imbalanced fermion gases.

Inspired by the rapid experimental progress, this line of research exploded with theoretical activity, leading to a wealth of predictions that continue to be explored in the laboratory. These include an extension of the $T=0$ microscopic analysis of the detuning-imbalance phase diagram [59, 66] to finite temperature [68, 69, 70, 71], proposals for numerous new phases and transitions, and an analysis of the strongly interacting normal state appearing at high imbalance [72, 73, 74]. The universal phenomenology of a balanced [75, 76, 177, 178, 79, 80] unitary Fermi gas has also been extended to a finite imbalance [81, 82, 83]. Along with these theoretical developments, new and powerful experimental probes (e.g., RF spectroscopy [72], in-situ density profile measurements [57], and measurements of collective modes [74]) have been brought to bear on this rich system, and in turn have stimulated further theoretical predictions. An overview of the considerable progress in the current understanding and of the 
remaining open questions in these imbalanced resonant Fermi gas systems is the focus of this review. Aimed to be significantly briefer and less comprehensive, it complements significantly more detailed recent reviews by Gurarie and Radzihovsky [20], by Bloch, Dalibard, and Zwerger [19], by Giorgini, Pitaevskii, and Stringari [21] and by Ketterle and Zwierlein [22].

The present review provides an overview of the current status of the field of imbalanced fermion gases, and is considerably broader than our recent research article [66] that only provided the details of our own theoretical calculations of the $T=0$ mean-field phase diagram. In this review, we aim to avoid the details of analyses while discussing the present-day understanding of the $T=0$ mean-field theory [59, 66] and the results of finite temperature mean-field and large- $\mathrm{N}$ calculations in Sec. 2, the nature and stability of strong fluctuations in the FFLO state in Sec. 3, the predictions of the local density approximation in Sec. 4, and the status of the most up to date experimental observations on this system in Sec. 5. In Sec. 6, we conclude with a few remarks about the future directions of this exciting subject in the physics of correlated fermions.

\subsection{Two-body Feshbach-resonant scattering}

Before turning to the analysis of states at finite density, we briefly review the Feshbachresonance mediated interaction, that (at finite density) leads to superfluidity in fermionic atomic gases. From a broad perspective, the low-energy atomic scattering and therefore (through the T-matrix) the effective inter-atomic attraction is strongly influenced by the presence of a bound (or quasi-bound) state in the two-atom potential. More microscopically, Feshbach resonant scattering in the "open" channel takes place when there is an energetically-nearby "closed" channel, that is weakly coupled to it by the hyperfine interaction. Crucially, due to a Zeeman splitting between the bound state (closed channel) and the scattered states (open channel), an external magnetic field can be used to change the bound-state energy relative to the continuum of the scattering atoms, and thereby tune the effective interactions between scattering atoms.

The Feshbach-resonant scattering of the open-channel fermions is characterized by the s-wave scattering amplitude $f_{0}(k)$, that by unitarity and analyticity [84] is required to take the form

$$
f_{0}(k)=\frac{1}{-a_{\mathrm{s}}^{-1}+\frac{1}{2} r_{0} k^{2}-i k},
$$

with $a_{\mathrm{s}}$ the scattering length and $r_{0}$ the effective range parameter that is negative in the resonant regime [20]. As the closed-channel bound-state energy is tuned through zero by adjusting an external magnetic field $B$, the scattering length diverges as

$$
a_{\mathrm{s}}=a_{b g}\left(1-\frac{B_{w}}{B-B_{0}}\right),
$$

with $a_{b g}$ the background scattering length, $B_{0}$ the resonance position (at which the bound-state energy vanishes) and $B_{w}$ the resonance width. 
A microscopically faithful way to capture the Feshbach resonance phenomenology is through the so-called two-channel model [35, 36, 27], in which the closed channel molecules appear explicitly in the Hamiltonian and give the scattering amplitude in Eq. (11). In practice, however, present-day experiments on superfluid Fermi gases can only access low energies (densities), that can be described by a simpler, more universal one-channel model to which the two-channel model reduces in the low-energy, $k\left|r_{0}\right| \ll 1$ limit [20, 85]. We thus focus on this experimentally relevant limit, described by the one-channel model Hamiltonian

$$
H=\sum_{\mathbf{k}, \sigma} \epsilon_{k} \hat{c}_{\mathbf{k} \sigma}^{\dagger} \hat{c}_{\mathbf{k} \sigma}+\frac{\lambda}{V} \sum_{\mathbf{k q p}} \hat{c}_{\mathbf{k} \uparrow}^{\dagger} \hat{c}_{\mathbf{p} \downarrow}^{\dagger} \hat{c}_{\mathbf{k}+\mathbf{q} \downarrow} \hat{c}_{\mathbf{p}-\mathbf{q} \uparrow},
$$

for the two species $(\sigma=\uparrow, \downarrow)$ of open-channel fermions created by the anticommuting operator $\hat{c}_{\mathbf{k} \sigma}^{\dagger}$, with the single-particle energy $\epsilon_{k}=\hbar^{2} k^{2} / 2 m$, mass $m$, and volume $V$. The attractive interactions are parameterized by $\lambda<0$.

Through a standard T-matrix scattering calculation, that gives $-f_{0}(k)=\frac{m}{4 \pi \hbar^{2}} T_{k}$, the pseudo-potential parameter $\lambda$ can be related to the experimentally determined, magnetic field dependent [25, 26] scattering length

$$
\frac{m}{4 \pi a_{\mathrm{s}} \hbar^{2}}=\frac{1}{\lambda}+\frac{1}{V} \sum_{\mathbf{k}} \frac{1}{2 \epsilon_{k}},
$$

where the ultraviolet-divergent second term is regularized by a microscopic momentum cutoff scale $\Lambda \sim 1 / d$ set by the closed-channel molecular extent $d$. This gives (with $\hbar=1$ throughout)

$$
\begin{aligned}
a_{\mathrm{s}}(\lambda) & =\left(\frac{4 \pi}{m \lambda}+\frac{2 \Lambda}{\pi}\right)^{-1} \equiv \frac{m}{4 \pi} \lambda_{R}, \\
& =\frac{m}{4 \pi} \frac{\lambda}{1-\lambda / \lambda_{c}}
\end{aligned}
$$

where $\lambda_{R}$ can be called the renormalized coupling and $\lambda_{c}=-\frac{2 \pi^{2}}{\Lambda m}$ is the critical coupling $\lambda$ at which the scattering length diverges. The above relation allows the definition of the model and therefore a reexpression of physical observables in terms of the experimentally defined (UV-cutoff independent) scattering length $a_{\mathrm{s}}$.

\subsection{Resonant Fermi gas at finite density: limits of validity}

In contrast to the two-body problem discussed above, a resonant many-body system at finite density presents a formidable challenge, that in most cases can only be treated approximately. Although many uncontrolled (but illuminating) approximations have appeared in the literature 34, 35, 36, 38] dating back to the pioneering works studying the BEC-BCS crossover [31, 32, 33], such one-channel model studies across the unitary point can only be trusted qualitatively. The reason is that the one-channel model at finite density $n$ exhibits only a single dimensionless gas parameter $n a_{\mathrm{s}}^{3}$ (or, equivalently, $k_{\mathrm{F}} a_{\mathrm{s}}$, with the Fermi momentum $k_{\mathrm{F}}$ ), a measure of the resonant interaction strength, that diverges upon approach to the Feshbach resonance, where $\left|a_{\mathrm{s}}\right| \rightarrow \infty$. Thus, a 
perturbative expansion in $k_{\mathrm{F}} a_{\mathrm{s}}$ is precluded sufficiently close to the resonance and is useful only far from the resonance, deep in the BCS and BEC limits, where $k_{\mathrm{F}}\left|a_{\mathrm{s}}\right| \ll 1$.

Nevertheless, trustworthy treatments of such strongly interacting, scale-invariant systems are indeed possible, taking a cue from the studies of critical phenomena [86, 87, 88, where analogous challenges have been surmounted almost 40 years ago. The approach is to "deform" the physical model to one that exhibits a mathematical small parameter, such as the deviation $\epsilon \equiv d_{u c}-d$ of spatial dimension $d$ from an upper-critical dimension, $d_{u c}$, above which the behavior is simple, or a $1 / N_{f}$ expansion about a large number of atom flavors, $N_{f}$, with the exactly solvable $N_{f} \rightarrow \infty$ limit formalizing and justifying earlier mean-field approximations. There has been considerable success in applying these field-theoretic methods to a unitary Fermi gas [82, 83, 89, 90]. These methods have also been embellished in uncontrolled "self-consistent" resummation schemes, that do not always lead to an improved description [91]. From the related renormalization group point of view, in the solvable vacuum (two particle) limit of the previous subsection, a Feshbach resonance with $\left|a_{\mathrm{s}}\right| \rightarrow \infty$ corresponds to a critical point [82, 83], that separates two distinct "phases" (vacua at zero density) on the BCS (positive detuning, $a_{\mathrm{s}}<0$ ) and BEC (negative detuning, $a_{\mathrm{s}}>0$ ) sides of the resonance. At a finite density $n$, this transition between the corresponding BCS and BEC paired superfluids is converted to a smoothed crossover, analogous to a paramagnetferromagnet crossover in a magnetic field.

Another useful approach to a controlled theory of the BEC-BCS crossover is through a narrow Feshbach resonance[27, 20], described by the two-channel model [23]. In the latter, in addition to the gas parameter $k_{\mathrm{F}} a_{\mathrm{s}}$, there appears another dimensionless parameter,

$$
\gamma=\frac{\sqrt{8}}{\pi} \sqrt{\frac{\Gamma_{0}}{\epsilon_{\mathrm{F}}}}=\frac{8}{\pi} \frac{1}{k_{\mathrm{F}}\left|r_{0}\right|},
$$

that is the dimensionless ratio of the width of the resonance, $\Gamma_{0}$ (proportional to $B_{w}$, set by the strength of the Feshbach resonance coupling, the hybridization amplitude of an atom-pair with a molecule) to the Fermi energy $\epsilon_{\mathrm{F}}$. This resonance-width parameter $\gamma$ naturally allows a distinction between wide $(\gamma \gg 1)$ and narrow $(\gamma \ll 1)$ resonances. Equivalently, these are contrasted by whether, upon growth near the resonance, the scattering length $a_{\mathrm{s}}(B)$ reaches the effective range $\left|r_{0}\right|$ first (the broad resonance) or the atom spacing $k_{F}^{-1} \sim n^{-1 / 3}$ first (the narrow resonance). A quantitatively-accurate description of the full BEC-BCS crossover, perturbative in $\gamma$, can thus be obtained for narrow Feshbach resonances, making them attractive from the theoretical point of view. The ability to treat narrow resonant systems perturbatively physically stems from the fact that such an interaction, although arbitrarily strong at a particular energy, is confined only to a narrow energy window around the resonance energy [27, 20].

In practice most $s$-wave Feshbach resonances studied to date are broad, $\gamma \gg 1$, with one notable exception discussed in Ref. [92. However, even for broad resonances (characteristic of a featureless attractive potential, where due to lack of a large potential barrier no long-lived resonant state exists at positive energy) $\gamma$ can be thought of as 
a mathematical expansion parameter about the solvable narrow resonance $(\gamma \rightarrow 0)$ limit, analogous to the $1 / N_{f}$ and $\epsilon$ expansions. Furthermore, because of the density dependence $\gamma \sim 1 / n^{1 / 3}$, in principle $\gamma$ can be reduced by working at higher atomic densities.

\subsection{Balanced BEC-BCS superfluid crossover}

Before turning to the main subject of polarized (imbalanced) resonant Fermi gases, we briefly review the salient points of a balanced gas and the associated BEC-BCS crossover. Since the physical interaction is everywhere attractive (although the scattering length changes sign), the ground state of the system is expected to be an s-wave superfluid for the full range of detuning. The latter will transition to a nonsuperfluid thermal state at a sufficiently high temperature $T_{c}$ (tunable via the FR), or to a Mott or Bose-glass insulator when subjected to a sufficiently strong commensurate periodic potential or quenched disorder [8].

The qualitative picture for the superfluid state across the resonance follows from the aforementioned solvable narrow resonance limit [27, 20], in which the closed channel molecule is long lived and can therefore be treated as an independent particle even for positive detuning. For detuning larger than twice the Fermi energy, closed-channel molecules are too energetically costly and most of the atoms are in the form of openchannel fermions, forming a weakly BCS-paired Fermi sea, with an exponentially small molecular density, induced by the weak Feshbach resonant coupling. The BEC-BCS crossover initiates as the detuning is lowered below an energy scale of order of $2 \epsilon_{\mathrm{F}}$, where a finite density of atoms begins to bind into Bose-condensed closed-channel molecules, stabilized by the Pauli principle. The resulting molecular (closed-channel) superfluid coexists with the strongly-coupled BCS superfluid of (open-channel) Cooper pairs, that, while symmetry-identical and hybridized with it by the Feshbach resonant coupling, is physically distinct from it. This is made particularly vivid in highly anisotropic, one dimensional traps, where the two distinct (molecular and Cooper-pair) superfluids can actually decouple due to quantum fluctuations, suppressing the Feshbach coupling at low energies 93. The crossover to the BEC superfluid terminates around zero detuning, where the conversion of open-channel atoms (forming Cooper pairs) into closed-channel molecules is nearly complete. In the asymptotic negative-detuning regime a true bound state appears in the closed-channel, leading to a positive scattering length and a twobody repulsion in the open-channel. In between, as the position of the Feshbach resonance is tuned through zero energy, the system is taken through (what would at zero density be) a strong unitary scattering limit, corresponding to a divergent scattering length, that is nevertheless quantitatively accessible in the narrow resonance limit, where $\gamma \sim 1 /\left(k_{F}\left|r_{0}\right|\right)$ plays the role of a small parameter.

Although the above features of the crossover are no longer expected to vividly appear in the experimentally relevant broad resonance limit, it is useful to keep this qualitative picture in mind. The detailed description of the broad resonance 


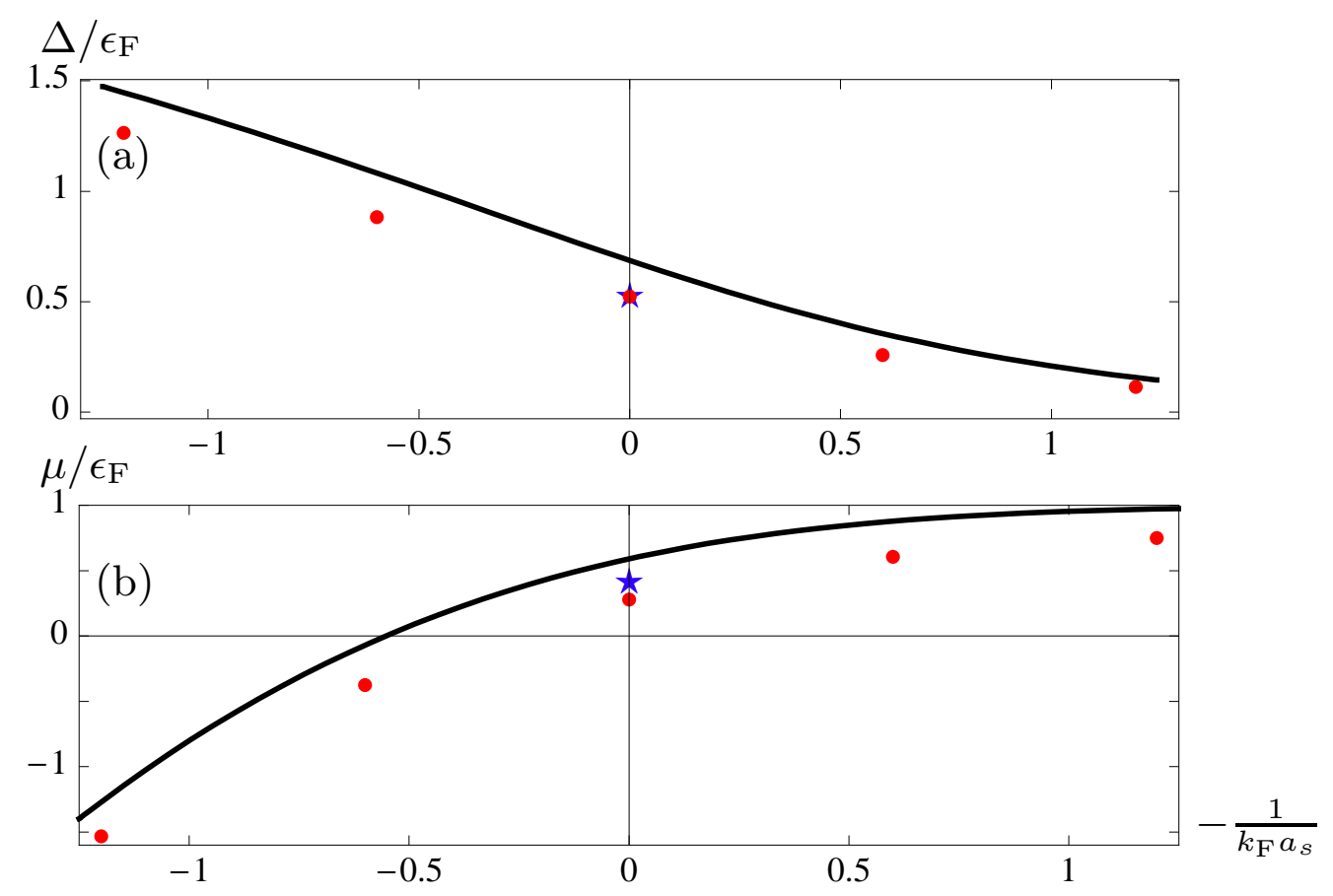

Figure 1. Order parameter $\Delta$ (panel a) and chemical potential $\mu$ (panel b) as a function of $-\left(k_{\mathrm{F}} a_{\mathrm{S}}\right)^{-1}$, with the BEC regime on the left and the BCS regime on the right. The solid black lines are the mean-field results for $\mu$ and $\Delta$, following Ref. 94]. The red circles include the $\mathcal{O}\left(1 / N_{f}\right)$ corrections [83, evaluated at $N_{f}=1$. The star symbols at unitarity are the results of the quantum Monte Carlo calculation of Ref. [77.

emerges when the Hamiltonian is treated in the mean-field approximation (although the aforementioned $\epsilon$ - and $1 / N_{f^{-}}$expansions can also be utilized to systematically correct it). At $T=0$, a standard mean-field analysis (that is exact in the large $N_{f}$ limit [82, 83]) of $H_{\mu}=H-\mu N$ gives the grand-canonical ground state energy density

$$
\begin{aligned}
E_{G} & =-\frac{\Delta^{2}}{\lambda}+V^{-1} \sum_{k}\left(\xi_{k}-E_{k}\right), \\
& =-\frac{m}{4 \pi a_{\mathrm{s}}} \Delta^{2}+\int \frac{d^{3} k}{(2 \pi)^{3}}\left(\xi_{k}-E_{k}+\frac{\Delta^{2}}{2 \epsilon_{k}}\right),
\end{aligned}
$$

with $\xi_{k} \equiv \epsilon_{k}-\mu$ and $E_{k}=\sqrt{\xi_{k}^{2}+\Delta^{2}}$, and in the second line $E_{G}$ was reexpressed in terms of the scattering length, thereby also leading to a finite pairing integral (the second term). A minimization of $E_{G}(\Delta, \mu)$ over the variational superfluid order parameter $\Delta$ ensures its expression in terms of the fermion pair $\Delta=\lambda\left\langle\hat{c}_{\downarrow}(\mathbf{x}) \hat{c}_{\uparrow}(\mathbf{x})\right\rangle$, i.e., gives the gap equation, and the chemical potential enforces the total fermion number equation $n=-\frac{\partial E_{G}}{\partial \mu}=V^{-1} \sum_{\mathbf{k}, \sigma}\left\langle c_{\mathbf{k} \sigma}^{\dagger} c_{\mathbf{k} \sigma}\right\rangle$.

The integrals in the mean-field ground-state energy Eq. (8), and the gap and number equations, can be expressed in terms of elliptic integrals [94], and the simultaneous numerical solution of the gap and number equations yields the mean-field $\Delta$ and $\mu$ as 
a function of the detuning parameter $-\frac{1}{k_{\mathrm{F}} a_{\mathrm{s}}}$, plotted as solid lines in Fig. 1. The red circles include the leading $1 / N_{f}$ correction, at $N_{f} \rightarrow 1$, following Ref. [83], while the star symbols at the unitary point are quantum Monte Carlo results [77].

It is also illuminating to obtain limiting analytical expressions to the mean-field results. In the BCS regime $\left(1<-\frac{1}{k_{\mathrm{F}} a_{\mathrm{s}}}\right), \mu>0$ and $\Delta \ll \mu$, one finds (defining $c=\frac{m^{3 / 2}}{\sqrt{2} \pi^{2}}$, with the three-dimensional density of states $N(\epsilon)=c \sqrt{\epsilon}$ ] [66]:

$$
E_{G} \approx-\frac{m}{4 \pi a_{\mathrm{s}}} \Delta^{2}-\frac{8 c}{15} \mu^{5 / 2}-c \sqrt{\mu} \Delta^{2}\left(\frac{1}{2}-\ln \frac{\Delta}{8 \mathrm{e}^{-2} \mu}\right)
$$

that is non-analytic in $\Delta$, reflecting the well-known fact that an arbitrary weak attraction yields a pairing instability. To leading order in $\Delta \ll \mu$, this gives the standard BCS expressions for the gap $\left(0=\frac{\partial E_{G}}{\partial \Delta}\right)$ and number $\left(n=-\frac{\partial E_{G}}{\partial \mu}\right)$ equations

$$
\begin{aligned}
& \Delta \approx \Delta_{\mathrm{BCS}} \equiv 8 \mathrm{e}^{-2} \epsilon_{\mathrm{F}} \exp \left(\frac{\pi}{2 k_{\mathrm{F}} a_{\mathrm{s}}}\right), \\
& \mu \approx \epsilon_{\mathrm{F}} .
\end{aligned}
$$

Here, the Fermi energy $\epsilon_{\mathrm{F}}$ is defined in terms of the particle density via $n=\frac{4}{3} c \epsilon_{\mathrm{F}}^{3 / 2}$. In the asymptotic negative-detuning BEC regime of $-\frac{1}{k_{\mathrm{F}} a_{\mathrm{s}}}<-1$, in which $\mu<0$ and $|\mu| \gg \Delta$, one instead obtains the analytic Landau form

$$
E_{G} \approx-\frac{m}{4 \pi a_{\mathrm{s}}} \Delta^{2}+\left.\frac{1}{2} c \sqrt{|\mu| \mid} \Delta\right|^{2}\left(\pi+\frac{\pi}{32} \frac{\Delta^{2}}{\mu^{2}}\right),
$$

that leads to the gap and number equations (for $\Delta \ll|\mu|$ )

$$
\begin{aligned}
\mu & \approx-\frac{1}{2 m a_{\mathrm{s}}^{2}}, \\
\Delta & \approx \epsilon_{\mathrm{F}} \sqrt{\frac{16}{3 \pi k_{\mathrm{F}} a_{\mathrm{s}}}},
\end{aligned}
$$

with $\mu$ equal to one-half the two-atom binding energy in the BEC limit.

As an aside, we note that expressions for the ground state energy, the chemical potential, and the order parameter (gap) are all well-defined in the unitary $a_{s} \rightarrow \infty$ limit, respectively given by universal forms

$$
E_{G}=0.5906 \frac{3}{5} n \epsilon_{\mathrm{F}}, \quad \Delta=0.6864 \epsilon_{\mathrm{F}}, \quad \mu=0.5906 \epsilon_{\mathrm{F}},
$$

all expressible in terms of the only energy (or equivalently, density $n=k_{F}^{3} / 3 \pi^{2}$ ) scale $\epsilon_{\mathrm{F}}$ of the problem, with the numerical prefactors approximations (to lowest order in the $1 / N_{f}$-expansion [82, 83]) for what are expected to be universal numbers characteristic of a unitary Fermi gas.

The above analysis can be generalized to finite temperature, giving the transition temperature $T_{c}$ to the normal state, at unitarity also scaling with $\epsilon_{\mathrm{F}}$ (but which becomes inaccurate in the deep-BEC limit [32]). A finite temperature also introduces an additional energy scale, at unitarity leading to the generalization of the above numerical prefactors to dimensionless universal scaling functions of $\epsilon_{\mathrm{F}} / T$. 


\section{Imbalanced resonant Fermi gas}

\subsection{Model}

With this background in mind the imbalance in the number of the two species is incorporated by introducing two distinct chemical potentials, $\mu_{\sigma}=\left(\mu_{\uparrow}, \mu_{\downarrow}\right)$ to impose separately conserved fermion numbers $N_{\sigma}=\left(N_{\uparrow}, N_{\downarrow}\right)$, or equivalently total fermion number $N=N_{\uparrow}+N_{\downarrow}$ and species imbalance $\Delta N=N_{\uparrow}-N_{\downarrow}$. The corresponding grand-canonical Hamiltonian $H_{\mu_{\sigma}}=H-\mu N-h \Delta N$ is

$$
\mathcal{H}=\sum_{\mathbf{k}, \sigma}\left(\epsilon_{k}-\mu_{\sigma}\right) \hat{c}_{\mathbf{k} \sigma}^{\dagger} \hat{c}_{\mathbf{k} \sigma}+\frac{\lambda}{V} \sum_{\mathbf{k q p}} \hat{c}_{\mathbf{k} \uparrow}^{\dagger} \hat{c}_{\mathbf{p} \downarrow}^{\dagger} \hat{c}_{\mathbf{k}+\mathbf{q} \downarrow} \hat{c}_{\mathbf{p}-\mathbf{q} \uparrow},
$$

with $\mu_{\uparrow}=\mu+h$ and $\mu_{\downarrow}=\mu-h$ related to the total-number chemical potential $\mu$ and species-imbalance chemical potential $h$ (an effective Zeeman energy). The imbalanced resonant Fermi gas thermodynamics as a function of $N, \Delta N, T, a_{\mathrm{s}}$, i.e., the extension of the BEC-BCS crossover to a finite imbalance can then be computed by a variety of theoretical techniques, including quantum Monte Carlo [63, 95], mean-field theory [66, 67, 65, 66, 68], the large- $N_{f}$ (fermion flavor) [82, 83, 90] and $\epsilon$ expansions [89]. By studying Eq. (17) we have already specialized to the wide Feshbach resonance limit. However, this problem can also be studied in a controlled narrow resonance regime by working with a two-channel model as done in Refs. [59, 68].

\subsection{Analysis at zero temperature}

We now outline the resulting picture (that is in qualitative agreement with experiments [55, 56]) using standard mean-field theory [66] in the broad-resonance limit. At zero temperature this amounts to a minimization of the mean-field ground-state energy $E_{G}$ with respect to the variational parameters (defined below) at fixed $\mu$ and $h$. The derivatives with respect to $\mu$ and $h$

$$
\frac{N}{V}=-\frac{\partial E_{G}}{\partial \mu}, \quad \frac{\Delta N}{V}=-\frac{\partial E_{G}}{\partial h},
$$

then respectively give the total atom number, $N=N_{\uparrow}+N_{\downarrow}$ and species imbalance, $\Delta N=N_{\uparrow}-N_{\downarrow}$, imposed in cold-atom experiments. Our variational ansatz assumes pairing of the form

$$
\Delta(\mathbf{x})=\Delta_{\mathbf{Q}} \mathrm{e}^{i \mathbf{Q} \cdot \mathbf{x}},
$$

with $\Delta_{\mathbf{Q}}$ and $\mathbf{Q}$ variational parameters (with $\mathbf{Q} \neq 0$ to allow for the possibility of the simplest Fulde-Ferrell-Larkin-Ovchinnikov (FFLO) [96, 97] type state). With this, one can derive the ground-state energy density $E_{G}\left(\Delta_{\mathbf{Q}}, \mathbf{Q}, \mu, h\right)$ following Ref. [66]

$$
\begin{aligned}
E_{G}= & -\frac{m}{4 \pi a_{\mathrm{s}}}\left|\Delta_{\mathbf{Q}}\right|^{2}+\int \frac{d^{3} k}{(2 \pi)^{3}}\left(\xi_{k}+\frac{Q^{2}}{8 m}-E_{k}+\frac{\Delta_{\mathbf{Q}}^{2}}{2 \epsilon_{k}}\right) \\
& +\int \frac{d^{3} k}{(2 \pi)^{3}}\left[E_{\mathbf{k}+} \Theta\left(-E_{\mathbf{k}+}\right)+E_{\mathbf{k}-} \Theta\left(-E_{\mathbf{k}-}\right)\right],
\end{aligned}
$$


with $\Theta(x)$ the Heaviside step function and where we defined

$$
\begin{aligned}
& E_{k} \equiv\left[\left(\xi_{k}+\frac{Q^{2}}{8 m}\right)^{2}+\Delta_{\mathbf{Q}}^{2}\right]^{1 / 2}, \\
& E_{\mathbf{k} \pm} \equiv E_{k} \mp\left(h-\frac{\mathbf{k} \cdot \mathbf{Q}}{2 m}\right),
\end{aligned}
$$

which can be solved for the stationary $\Delta_{\mathbf{Q}}$ and $\mathrm{Q}$ by supplementing the gap equation with the condition

$$
\frac{\partial E_{G}}{\partial Q}=0
$$

Although the mean-field approximation is expected to be quantitatively inaccurate in the strongly-interacting unitary regime, it does provide a consistent qualitative picture and a natural starting point for more accurate theoretical methods.

An important point to keep in mind, for a proper analysis of $E_{G}\left(\Delta_{\mathbf{Q}}, \mathbf{Q}, \mu, h\right)$, is that not every simultaneous solution of the gap and number equations corresponds to a physical ground state of the system; the key additional criterion is that the solution $\Delta_{\mathbf{Q}}$ must also be a minimum of $E_{G}$ at fixed $\mu, h$. The verification that an extremum solution is indeed a minimum is particularly essential when (as is the case for a polarized Fermi gas) there is a possibility of a first-order transition, where $E_{G}$ exhibits local maxima and saddle points that separate its local minima. By working instead directly with the gap equation and imposing fixed $N$ and $\Delta N$ from the start, several authors have been lead to qualitatively and/or quantitatively incorrect phase diagrams [65, 98, 69], mistakenly associating local minima, saddle-points and/or even maxima with a ground state, and misidentifying first-order phase boundaries with the spinodals, as discussed in Refs. 67, 99].

Therefore, in our experience the safest path to the correct phase diagram is to work in the grand-canonical ensemble, use the global minimum of the free energy to map out the phase diagram at fixed $\mu, h$, and only then constrain it to the imposed total number and imbalance densities; the in-satisfiability of this final constraint is a signature of phase separation, resolved by an inhomogeneous coexistence of two phases in proportions imposed by the two density constraints.

\subsection{Phase diagram}

2.3.1. Zero temperature With these cautionary remarks in mind, as detailed in Ref. 66] $E_{G}\left(\Delta_{\mathbf{Q}}, \mathrm{Q}, \mu, h\right)$ can be analyzed analytically in a number of asymptotic regimes and numerically throughout. The result of its minimization directly leads to the zerotemperature phase diagram [66] at fixed density and chemical potential difference in Fig. 2 and at fixed density and imbalance in Fig. 3. The BCS regime of both figures is shown in Fig. 4 .

The qualitative features of the phase diagram can be readily understood. At sufficiently small Zeeman energy $h$ the fully-paired $P=0$ superfluid state (that has $\mathrm{Q}=0$, with $\left.\Delta_{\mathbf{Q}=0}=\Delta=\Delta_{B E C-B C S}\right)$ is stable across the full BEC-BCS crossover. As 


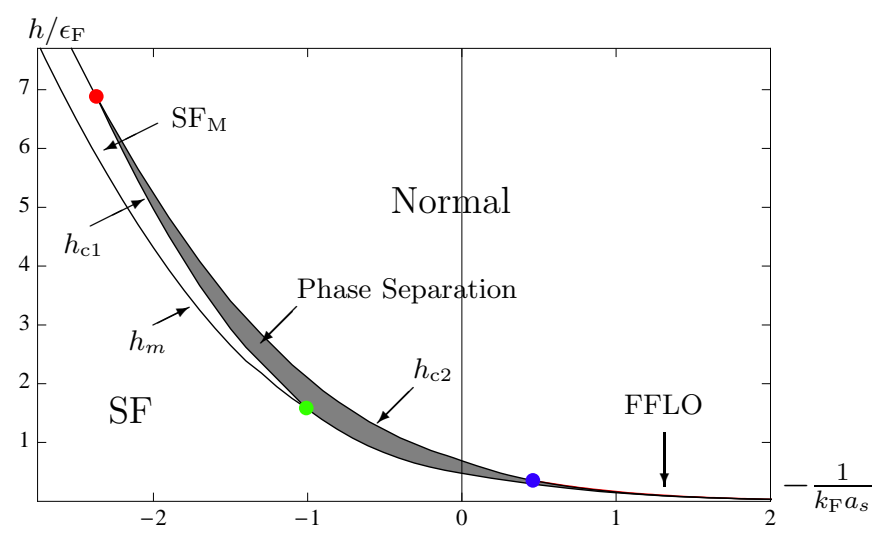

Figure 2. Mean-field phase diagram of imbalanced Fermi gases, as a function of inverse scattering length and chemical potential difference (Zeeman energy) $h$, showing regimes of an imbalanced magnetized superfluid $\left(\mathrm{SF}_{\mathrm{M}}\right)$ bounded by $h_{m}$ and $h_{\mathrm{c} 1}$, and a strongly-interacting normal Fermi liquid. The region denoted $S F$ is the balanced BEC-BCS crossover superfluid phase. The central shaded region is a regime of phase separation, where the gas inhomogeneously coexists between two phases. The FFLO region is indicated by an arrow and bounded, to the right of the blue point by $h_{\mathrm{FFLO}}$ and $h_{\mathrm{c} 2}$; however it is too small to be seen on this scale. A zoom-in of this region is shown in Fig. [4. The red point at $(-2.37,6.89)$ is a quantum tricritical point (QTP) separating the first- and second-order transitions from the $\mathrm{SF}_{\mathrm{M}}$ to the polarized Normal phase. The green point at $1 /\left(k_{F} a\right) \approx 1.01, h \approx 1.5$ is a critical end-point.

first shown by Sarma [51], in the BCS regime the paired state cannot accommodate any polarization (except for the $Q \neq 0 \mathrm{FFLO}$ state, which we shall neglect for the moment). This follows because the BCS s-wave paired state involves pairing at the Fermi surface and is fully gapped. However, in the presence of a population imbalance, the noninteracting Fermi surfaces have different volumes, determined by the Fermi momenta $k_{\mathrm{F} \uparrow}$ and $k_{\mathrm{F} \downarrow}$, disrupting pairing. With increasing $h$ and at fixed $\mu$, then, the BCS state undergoes a first-order transition to an unpaired magnetized normal state. At imposed average density (atom number $N$ ) and fixed $h$, this leads to phase separation for $h>h_{c 1}$, as shown in Fig. 2. Since the polarization only becomes nonzero for $h>h_{c 1}$ in the BCS regime, it is clear that, at fixed polarization $P$, one finds phase separation at arbitrarily small $P$, as shown in Fig. 3. Thus, an addition of excess spin- $\uparrow$ atoms to the paired BCS state leads to their spatial phase separation from the paired gas. In contrast, as illustrated in Fig. 3, in the deep BEC regime the imposed polarization is accommodated by a transition of the $P=0$ molecular superfluid to a polarized superfluid, $\mathrm{SF}_{\mathrm{M}}$, that is a homogeneous ground state of condensed molecules and a single-species (fully-polarized) Fermi gas.

The low- $h$ stability of the BEC-BCS superfluid is limited by the lower-critical Zeeman field, that in the weakly-paired BCS regime $\left(-1 / k_{\mathrm{F}} a_{\mathrm{s}} \gg 1\right)$ is of the order $h_{c 1} \sim \Delta_{\mathrm{BCS}} / \sqrt{2}$, exponentially small in $1 / k_{\mathrm{F}} a_{\mathrm{S}}$ (this is the Clogston limit [50]). We define $h_{c 1}$ to be the Zeeman field above which the system phase separates. With decreasing detuning, it grows to $h_{c 1} \sim \epsilon_{\mathrm{F}}$ around the unitary crossover regime $\left(-1<-1 / k_{\mathrm{F}} a_{\mathrm{s}}<1\right)$. 


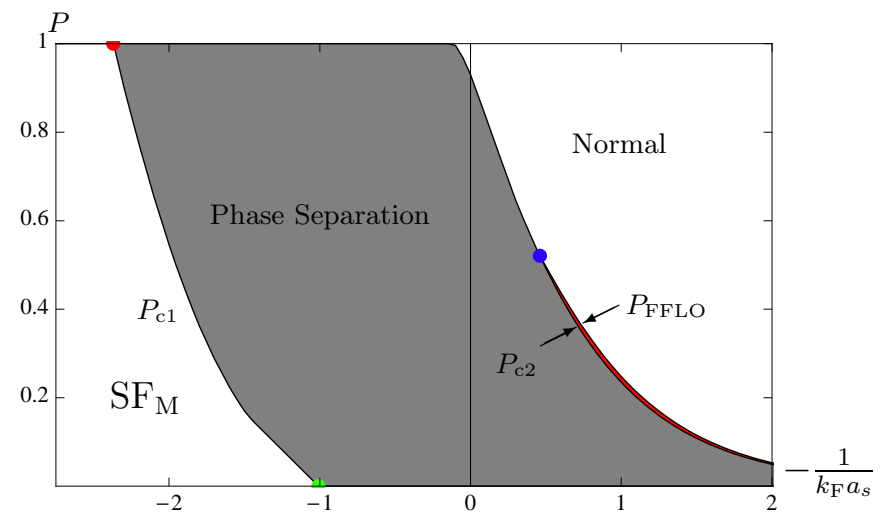

Figure 3. Mean-field phase diagram of imbalanced Fermi gases, as a function of inverse scattering length and imbalance $P$, showing regimes of magnetized (imbalanced) superfluid $\left(\mathrm{SF}_{\mathrm{M}}\right)$, FFLO (shaded red, bounded by $P_{\mathrm{FFLO}}$ and $\left.P_{\mathrm{c} 2}\right)$ and normal Fermi liquid. The central shaded region is a regime of phase separation, and the red point at $(-2.37,1)$ is a quantum tricritical point (QTP). The FFLO phase is only stable to the right of the blue point. The green point at $1 /\left(k_{F} a\right) \approx 1.01, P=0$ is a critical end-point.

Deep in the BEC regime $\left(1 / k_{\mathrm{F}} a_{\mathrm{S}} \ll-1\right)$, the stability of the BEC-BCS superfluid is instead controlled by the molecular binding energy $h_{m} \sim E_{b} \sim 1 / m a_{\mathrm{s}}^{2}$.

In the opposite limit of large Zeeman field, $h>h_{c 2}$ (at fixed $\left.N\right)$, a normal (unpaired) state is clearly stable, with $h_{c 2}$ exponentially small (but larger than $h_{c 1}$ ) in the BCS limit. With decreasing detuning, $h_{c 2}$ grows through $\sim \epsilon_{\mathrm{F}}$ in the crossover regime and increases further with the binding energy (while satisfying $h_{m}<h_{c 1}<h_{c 2}$ ) deeper in the BEC regime. The high field-induced depaired state is a partially (two Fermi surfaces) or fully (single Fermi surface) polarized normal Fermi liquid, $\Delta=0, P \neq 0$, respectively depending on the Zeeman field strength $h$ and the interaction strength $1 / k_{F} a$. Deep in the BEC regime this normal state is fully polarized, characterized by a single-species Fermi surface.

In the deep BCS regime, the interactions are weak and the large $h$ depaired state is clearly a weakly renormalized polarized Fermi liquid, while in the deep BEC regime the only depaired state is fully polarized $(P=1)$, that is, therefore, noninteracting. In contrast, in the crossover regime $\left(-1<-1 / k_{\mathrm{F}} a_{\mathrm{s}}<1\right)$ the interactions are resonant and strong, and therefore cannot be treated perturbatively. Nevertheless one can explicitly demonstrate using many-body $1 / N_{f}$-expansion techniques, that (earlier suggestions based on RF spectroscopy notwithstanding [72]) this depaired state remains a normal Fermi liquid, albeit a strongly renormalized one [90]. For example, at unitarity and $P \rightarrow 1^{-}$it is characterized by a suppressed quasi-particle residue $Z=0.47$, an enhanced effective mass $m_{*} \approx 1.8 m$, and a downward chemical potential shift $\delta \mu_{\downarrow}=-1.46 \epsilon_{\mathrm{F}}$.

In the presence of a population imbalance, another possible ground state is the FFLO phase, in which atoms pair at a nonzero center of mass momentum $Q \simeq k_{\mathrm{F} \uparrow}-k_{\mathrm{F} \downarrow}$ to accommodate the spin- $\uparrow$ and spin- $\downarrow$ Fermi-surface mismatch. Within mean-field theory, and assuming the simplest FFLO-type state, Eq. (19), in the BCS regime the 
above-mentioned first-order transition with increasing $h$ is into the FFLO phase. With further increase in $h$ the FFLO phase undergoes a continuous transition into the normal phase yielding the phase diagram at fixed $h$ and fixed density shown in Fig. $4 a$, and the phase diagram at fixed $P$ and fixed density shown in Fig. $4 \mathrm{~b}$. We observe that the FFLO phase is favored only for a thin sliver of $h$ or $P$, and for sufficiently weak interactions $k_{\mathrm{F}}\left|a_{\mathrm{s}}\right|<2$ in the BCS regime. In Sec,3, we discuss FFLO-type states in more detail and the strong effect of fluctuations on them.

Turning to the BEC regime, as illustrated in Fig. 2, at large negative detuning $\left(-1 / k_{\mathrm{F}} a_{\mathrm{s}}<-1\right)$ and at intermediate Zeeman fields, $h_{m}<h<h_{c 1}$, an imposed species imbalance can be accommodated by a homogeneous polarized (imbalanced) superfluid state, $\mathrm{SF}_{\mathrm{M}}$ (that we originally dubbed "magnetized superfluid" [59])[100]. This is not surprising as in this regime of strong attractive interactions pairing takes place in real space and a nonzero imbalance corresponds to the addition of a low density of excess fermions to a fluid of tightly-bound molecular bosons. As discussed in Ref. [101, this is analogous to the well-studied problem of ${ }^{3} \mathrm{He}-{ }^{4} \mathrm{He}$ mixtures (and, generally, bosonfermion mixtures [102, 103]), with fermionic ${ }^{3} \mathrm{He}$ atoms corresponding to the excess spin- $\uparrow$ fermions and known to exhibit a homogeneous phase (miscible in ${ }^{4} \mathrm{He}$ ) below a concentration of about $7 \%($ at $T=0)$ [104, 105].

In the simplest approximation, the $\mathrm{SF}_{\mathrm{M}}$ phase is characterized by a ground state that is the product of a fully polarized majority Fermi sea and a diatomic molecular condensate. This phase is therefore quite novel, as it is a hybrid state displaying stronglypaired superfluidity and gapless fermionic excitations around a single polarized Fermi surface, and is thereby expected to display features (e.g., linear low-temperature heat capacity, superflow, etc.) characteristic to both. The $\mathrm{SF}_{\mathrm{M}}$ is separated from the balanced $P=0$ superfluid by a continuous transition at $h=h_{m}$, the latter set by the binding energy.

However, as illustrated in Figs. 2 and [3, over the large shaded portion of the phase diagram, at intermediate detuning and Zeeman field (and polarization), it is not possible to accommodate a species number imbalance with a homogeneous, single component global minimum of $E_{G}$. This happens whenever two competing groundstate minima become degenerate, and are characterized by two distinct densities and polarizations for the same critical values of chemical potentials. Thus, as is standard for first-order transitions, it is not possible for the system to achieve a homogeneous state with intermediate values of the imposed densities [59, 66]. For the corresponding range of parameters in the shaded region the system thus phase separates [61] into two coexisting degenerate ground states with different densities in chemical equilibrium, such that the total imposed number and polarization constraints are satisfied. The resulting phase-separated state can be explicitly accounted for by generalizing the ground-state ansatz to include the possibility of such an inhomogeneous mixture [66].

One feature of the above phase diagram that has attracted recent attention is the quantum tricritical [106] point (QTP) [68, 66], appearing at $-1 /\left(k_{\mathrm{F}} a_{\mathrm{s}}\right) \simeq-2.37$ in the mean-field approximation and denoted as a red point Fig. 3. It separates a first-order 
(to the right) and second-order (to the left) $P$-increasing transition out of the polarized superfluid, $\mathrm{SF}_{\mathrm{M}}$. Upon decreasing $P$ from unity, the QTP separates two possible fates of an added spin-down fermion immersed in the polarized Fermi sea: To the left of the QTP, the magnetized superfluid phase appears, as added spin-down atoms form tightly-bound pairs with spin-up atoms, miscible with the remaining spin-up Fermi sea. In contrast, to the right of the QTP, the added spin- $\downarrow$ atoms will form molecular pairs, that are immiscible with the remaining majority Fermi sea (leading to phase separation to the right of the QTP). The third possibility for the added spin- $\downarrow$ atoms is to form a spin- $\downarrow$ Fermi sea. This is realized to the right in the phase diagram, beyond $-\frac{1}{k_{\mathrm{F}} a_{\mathrm{s}}} \simeq-0.1$, as seen in Fig. 3, where the phase separation region meets the normal Fermi liquid region at $P \rightarrow 1$.

A similarly interesting region of the phase diagram is the point (marked green in Figs. 2 and 3) at which the $\mathrm{SF}_{\mathrm{M}}$ state emerges (a critical end point, at which a line of second order transitions terminates at a first-order transition). This point is located on the $P=0$ axis, at $-1 /\left(k_{\mathrm{F}} a_{\mathrm{s}}\right) \simeq-1.01$ in the mean-field approximation and separates first- and second-order transitions, with increasing $P$, out of the fully paired, balanced BEC-BCS superfluid.

The above outlined mean-field phase diagram is in qualitative agreement with recent QMC calculations [95], with the quantitative discrepancies primarily traceable to the neglect, within mean field theory, of normal state interactions [107. The latter lower the normal state free-energy and corresponding $P_{c 2}$ below their mean-field values. Indeed, recent experiments [108, 109] have observed the $P_{c 2}$ phase boundary (but not the FFLO phase) in qualitative agreement with Fig. 3. finding, at unitarity, $P_{c 2} \simeq 0.3-0.36$ (Fig.2 in Ref. [109]) significantly below its mean-field prediction of $P_{\mathrm{c} 2}^{m f} \simeq 0.93$, but quite close to the result of the large- $N$ expansion [83] of $P_{\mathrm{c} 2}^{\text {largeN }} \simeq 0.302$ and the QMC result 95] of $P_{\mathrm{c} 2}^{Q M C} \simeq 0.4$.

However, experiments have not yet detected the $P_{\mathrm{c} 1}$ boundary separating the $\mathrm{SF}_{\mathrm{M}}$ and phase separation regimes, although the finite-temperature $\mathrm{SF}_{\mathrm{M}}$ phase has been observed; mean-field theory and QMC predict similar values for $P_{\mathrm{c} 1}$. To compare, we note that the tricritical point at $P=1$ occurs at $\left(k_{\mathrm{F}} a_{\mathrm{s}}\right)^{-1}=2.37$ in MFT and $\left(k_{\mathrm{F}} a_{\mathrm{s}}\right)^{-1}=2.14$ within QMC [95]. Additionally, the onset of the $\mathrm{SF}_{\mathrm{M}}$ phase occurs at $\left(k_{\mathrm{F}} a_{\mathrm{S}}\right)^{-1}=1.01$ in MFT and at $\left(k_{\mathrm{F}} a_{\mathrm{s}}\right)^{-1}=0.53$ within QMC [95].

One regime in which the problem simplifies (while still exhibiting strong-correlation effects) is the extreme polarized limit $P \rightarrow 1^{-}$of a small density of spin- $\downarrow$ atoms in a Fermi sea of spin- $\uparrow$ atoms, with the limiting case of one "impurity" spin- $\downarrow$ atom immersed in the majority (spin- $\uparrow$ ) Fermi sea. This problem has been studied using variational wavefunction [110, 111] and Monte Carlo [112] techniques, finding that one spin- $\downarrow$ Fermi atom interacts strongly with the spin- $\uparrow$ Fermi sea (forming a "polaron"), as reflected in values of the quasiparticle residue $Z$, effective mass $m_{*}$ and chemical potential $\delta \mu_{\downarrow}$ that deviate from that of free fermions. These polarons have been subsequently observed in experiments studying the $P \rightarrow 1^{-}$limit, obtaining (at unitarity) $Z \approx 0.39$ [73], $m_{*} \approx 1.17 m$ [74], and $\delta \mu_{\downarrow} \approx-0.64 \epsilon_{\mathrm{F}}$ [73], values that are in good agreement with QMC 


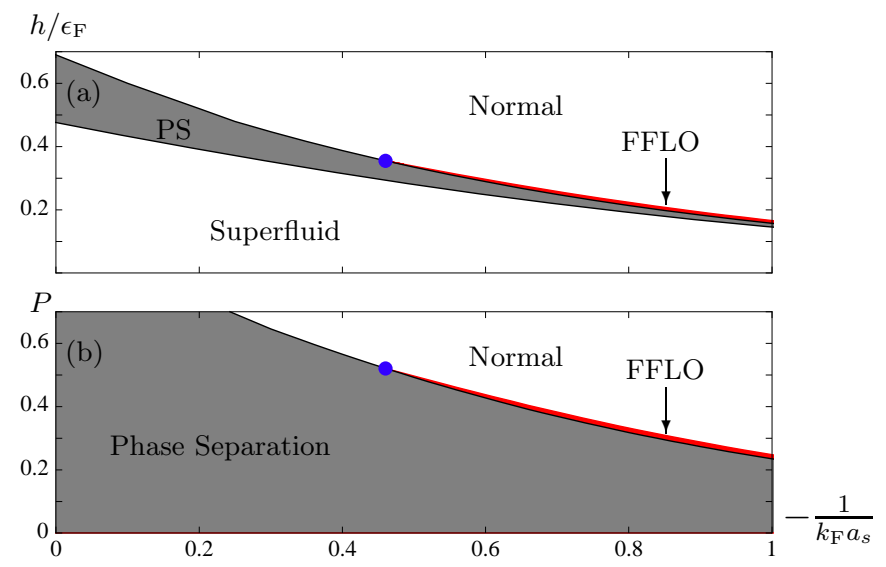

Figure 4. Panel a is a zoom-in of the phase diagram of Fig. 2 at fixed $h$ and inverse scattering length. Panel $\mathrm{b}$ is a zoom-in of the phase diagram of Fig. 3 at fixed $P$ and inverse scattering length. The horizontal axes are the same in the two panels. In each, the FFLO phase is only stable to the right of the blue point (as in Figs. 2 and 3).

and variational theories. Upon increasing the strength of the attractive interactions into the BEC regime, around $1 / k_{F} a \approx 1.27$ a phase transition is predicted [113] from a polaron-like state of the minority atom dressed by the majority Fermi sea (characterized by a nonzero fermionic pole with a finite residue $Z<1$ ) to a bosonic molecule dressed by the (majority) polarized Fermi sea and characterized by a vanishing residue of the fermionic pole $(Z \rightarrow 0)$. The properties of the latter state have been recently analyzed by a molecular variational wavefunction [113, 114, thereby constructing a phase diagram analogous to Fig. 2.

In addition to the basic (and well accepted) qualitative features of the imbalanced resonant paired superfluid, summarized by Figs. 2,3, and 4, a number of additional phases have been suggested that are not captured by the simplest mean-field ansatz. These include breached pair phases [60], deformed Fermi surface (nematic) phases [115, 116], possible further instabilities of the fully-polarized Fermi sea of the $S F_{\mathrm{M}}$ phase [101], and a number of more exotic fluctuation-driven FFLO-type phases that we discuss in Sec. [3.

2.3.2. Non-zero temperature Soon after the zero-temperature phase diagram in Fig. 3 was established [59, 66], the analysis was generalized to a finite temperature 68, 69, 70, 71, as illustrated in Fig.5. As usual, the finite-temperature thermodynamics and the corresponding phase diagram emerges from the minimization of the free energy $F$, that for the imbalanced resonant Fermi gas (because of a number of possible extrema) must be done with care similar to that of the zero-temperature case. While the $T=0$ phase diagram is largely established (despite an ongoing search for the FFLO phase, remaining questions about its extent to smaller imbalance, and the need for more detailed studies of the $S F_{M}$ ), the status of the finite- $T$ phase diagram in the strongly-interacting regime is more uncertain. The basic reasons for this can be traced to the original analysis by Nozieres and Schmitt-Rink [32] on the finite temperature balanced paired Fermi gas and 


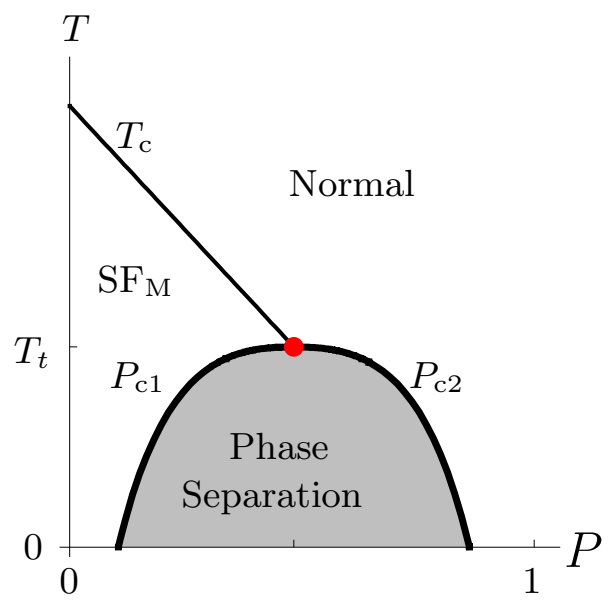

Figure 5. A schematic phase diagram for fixed detuning, illustrating an extension of the ground-state phase diagram, Fig. 3, to finite $T$, supported by theoretical calculations in the narrow-resonance regime 68. The red dot is a tricritical point. For $-\frac{1}{k_{\mathrm{F}} a_{\mathrm{s}}}>-2.37$, to the right of the QTP, the phase boundaries $P_{\mathrm{c} 1}$ and $P_{\mathrm{c} 2}$ extend to finite $T$, merging at a tricritical point. In the regime where the FFLO can be stable, one expects a sliver of FFLO phase along the $P_{\mathrm{c} 2}$ boundary. The schematic phase diagram depicts a putative regime of detuning, where at $T \rightarrow 0, P_{\mathrm{c} 1}>0$ and $P_{\mathrm{c} 2}<1$. Such a regime does not appear within the mean-field approximation, but is found in QMC calculations 95 .

its BEC-BCS crossover. While the simple BEC-BCS variational mean-field wavefunction gives a reasonable approximation for $T=0$ properties across the phase diagram, for the gas at finite temperature one must include the effect of thermally excited molecular pairs and fermionic single-particle excitations to obtain a reasonable $T_{\mathrm{c}}$. Because these excitations are expected to be strongly interacting around the unitary regime (FR point) a quantitatively trustworthy description remains a challenging open problem for both balanced and imbalanced gases.

However, quantitative predictions for phase boundaries notwithstanding, it is likely that the form of the finite-T phase diagram, predicted by Ref. [68] via a controlled narrow resonance two-channel model analysis [27, 20, 59, 66] is qualitatively correct. The finite- $T$ phase diagram, illustrated Fig. 5 is reminiscent of the phase diagram of ${ }^{3} \mathrm{He}-{ }^{4} \mathrm{He}$ mixtures.

A number of new ingredients emerge at finite temperature. Because (in contrast to the fully-gapped BEC-BCS paired ground state) "spin"-ful quasi-particles can carry a finite imbalance, at finite temperature a chemical potential imbalance $h$ always induces a nonzero species polarization. Thus at finite $T$ the BEC-BCS singlet superfluid state will no longer be distinguished from the $\mathrm{SF}_{\mathrm{M}}$ by a vanishing polarization. Therefore at nonzero temperature $h_{m}$ is a crossover, that at low temperature is expected to remain relatively sharp. On the BCS side of the resonance at low $T$ the first-order transition out of the paired BCS superfluid is expected to survive, though likely to reduce in strength. 
Another finite temperature effect is the migration of the zero-temperature tricritical point of Fig. 33into the $P<1$ regime [68, 70, 117, as illustrated in Fig. 15. Finally, as we will discuss below, a strikingly more significant qualitative effect of thermal fluctuations is predicted to arise in the putative unidirectional FFLO states[126]. The enhanced role of fluctuations in such periodically paired superfluids is associated with underlying rotational and translational invariance that is spontaneously broken. As we will discuss in more detail in the next section, fluctuations lead to universal power-law density correlations in the LO state, and a melting of the translational order (that can liberate fractional charge dislocations and vortices) and induce quantum nematic phases.

\section{Fulde-Ferrell-Larkin-Ovchinnikov superfluid}

One of the more interesting alternatives to the resolution of the species-imbalance imposed frustration is the Fulde-Ferrell-Larkin-Ovchinnikov (FFLO) [96, 97] superfluid. In this state the Fermi surface mismatch is accommodated by finite momentum pairing, that in its most generic form is a paired supersolid, that is somewhat of a holy grail in condensed matter physics. First proposed more than 45 years ago, this enigmatic paired state has been explored extensively in the context of solid state superconductors and high-energy physics systems [43, 44]. However, with the exception of recent promising experiments on $\mathrm{CeCoIn}_{5}[118$, , 119], the FFLO state has so far eluded definitive observations.

\subsection{Theoretical description}

The existence of the FFLO state is most easily detected, theoretically, through a transition from the polarized normal state upon reduction of the chemical potential difference, $h$ (or equivalently, by reduction of the species imbalance). Assuming (and verifying a posteriori) a continuous (or weakly first-order) N-FFLO transition, a perturbative treatment via a Landau expansion in the pairing order parameter $\Delta_{\mathbf{Q}}$, defined by $\Delta(\mathbf{x})=\sum_{\mathbf{Q}_{n}} \Delta_{\mathbf{Q}_{n}} e^{i \mathbf{Q}_{n} \cdot \mathbf{x}}$, is justified. Standard analysis gives Landau free energy density

$$
\mathcal{H}_{G L}=\sum_{\mathbf{Q}} \Delta_{\mathbf{Q}}^{*} \varepsilon_{Q} \Delta_{\mathbf{Q}}+\sum_{\mathbf{Q}_{1}, \mathbf{Q}_{2}, \mathbf{Q}_{3}} v_{\mathbf{Q}_{1}, \mathbf{Q}_{2}, \mathbf{Q}_{3}, \mathbf{Q}_{4}} \Delta_{\mathbf{Q}_{1}}^{*} \Delta_{\mathbf{Q}_{2}} \Delta_{\mathbf{Q}_{3}}^{*} \Delta_{\mathbf{Q}_{4}}+\ldots,
$$

where translational invariance constrains $\varepsilon_{Q}$ to be a function of the magnitude of $\mathbf{Q}$ only, $\mathbf{Q}_{4}=\mathbf{Q}_{1}-\mathbf{Q}_{2}+\mathbf{Q}_{3}, \Delta_{\mathbf{Q}}$ is a Fourier transform of $\Delta(\mathbf{x})$, and

$$
\varepsilon_{Q} \approx \frac{3 n}{4 \epsilon_{\mathrm{F}}}\left[-1+\frac{Q^{2}}{2 \gamma \mu}+\frac{1}{2} \ln \frac{v_{F}^{2} Q^{2}-4 h^{2}}{\Delta_{\mathrm{BCS}}^{2}}+\frac{h}{v_{F} Q} \ln \frac{v_{F} Q+2 h}{v_{F} Q-2 h}\right],
$$

obtained either by a direct Landau expansion [97] or via expansion of the Bogoliubov-de Gennes $(\mathrm{BdG})$ equation [66] to quadratic order in $\Delta_{\mathbf{Q}}$. Simple analysis shows that $\varepsilon_{Q}$ has a minimum at $|\mathrm{Q}| \approx 1.81 \Delta_{\mathrm{BCS}} / \hbar v_{F} \approx 0.58 / \xi_{0}$, infinitely degenerate with respect to the detailed nature of the FFLO state, thereby leaving the quartic interactions to lift this degeneracy, i.e., to select the set of Q's defining a specific type of the FFLO state. 
As first demonstrated by LO, just below $h_{c 2}$ it is the $\pm Q$ periodic (cosine) state that is selected.

However, the above treatment cannot be justifiably extended far below $h_{c 2}$, down to the transition to the uniform fully-paired superfluid state near $h_{c 1}$. There, the order parameter is no longer small and therefore must be treated nonperturbatively. Namely, a complete functional dependence of $\mathcal{H}[\Delta(\mathbf{x})]$ may need to be taken into account, and an arbitrary form of $\Delta(\mathbf{x})$ (arbitrary set of Fourier harmonics $\Delta_{\mathbf{Q}}$ ) must be considered.

The findings of LO [97 motivate one to focus on a unidirectional FFLO order characterized by a colinear set of the $\mathbf{Q}_{n}$. Under this restriction, FFLO states fall into two, LO and FF universality classes. The LO (FF) states are characterized and distinguished by breaking (preserving) translational and preserving (breaking) timereversal symmetries. The low-energy properties of such states can be well captured with a single $\mathrm{Q}$ pair ( $\mathrm{LO}$ ) and a single $\mathrm{Q}$ wave vector $(\mathrm{FF})$ approximations, as we describe below. However, the analysis of energetics of these states far below $h_{c 2}$ requires the aforementioned fully nonlinear analysis.

In principle this can be accomplished through a BdG treatment. However, its analytical solution (outside 1d) is only tractable for a single $\mathbf{Q}$ FF state. This approach predicts a narrow sliver of $\mathrm{FF}$ state near $h_{c 2}$, that closes down at $-1 / k_{\mathrm{F}} a_{\mathrm{s}} \approx 0.46$, as the strongly coupled regime is entered [59, 66]. The most direct interpretation of this finding is reflected in our $T=0$ phase diagram in Fig. 3159, 66, and is quite pessimistic for the observation of any of the FFLO states due to its narrow range of stability and the trap induced cloud inhomogeneity.

However, an alternative, more optimistic scenario is suggested by early numerical treatments of $2 \mathrm{~d}$ and $3 \mathrm{~d}$ BdG equations [120, 121], exact 1d solution [122], density functional theory results [123], and recent findings of a negative domain-wall energy in an otherwise uniform singlet BCS superfluid[124, 125]. These fully nonlinear (in $\Delta(\mathbf{x}))$ findings support the idea that a LO-type periodic superfluid state of domainwalls (corresponding to a large set of colinear wavevectors in $\Delta(\mathbf{x})$ ) may be significantly more stable down to the vicinity of $h_{c 1}$. The LO state can be thought of as a periodically ordered micro-phase separation between the normal and BCS states, that thus naturally replaces the macro-phase separation ubiquitously found in the BEC-BCS detuningpolarization phase diagram. In Fig. 6, we show a proposed phase diagram, assuming that the SF-LO phase transition is indeed continuous and occupies a wider regime of the phase diagram than the mean-field prediction (see Ref. [125]).

Despite the extended history of FFLO states, only recently has the role of quantum and thermal fluctuations been understood[126]. This study developed Goldstone mode models for the LO and FF universality classes and analyzed the corresponding beyondmean-field phenomenology. Focusing on the more stable LO state [97, 120, 121, 125, 124, it was demonstrated that in contrast to the conventional (uniform) superfluid and FF states, the LO superfluid exhibits two Goldstone modes, $\theta_{ \pm Q}$, corresponding to two primary order parameters $\Delta_{ \pm \mathbf{Q}}$ (with phases for high harmonics locked to these two). Equivalently, low-energy fluctuations are characterized by the superfluid phase, 

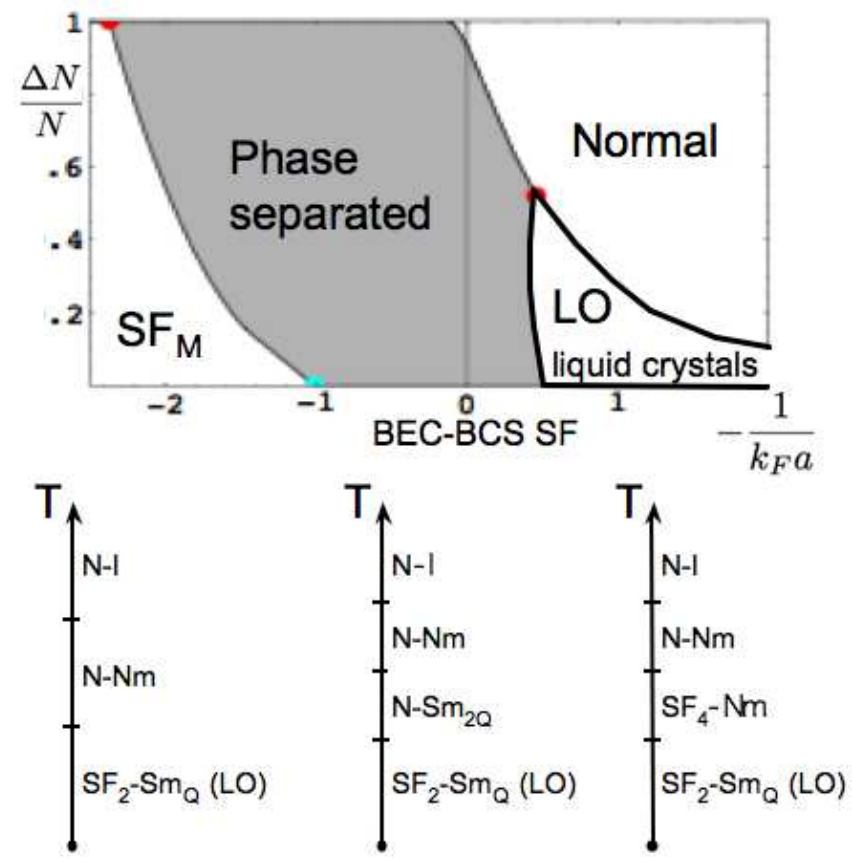

Figure 6. Proposed schematic phase diagram of $\Delta N / N$ vs. $1 /\left(k_{\mathrm{F}} a_{\mathrm{s}}\right)$, showing LO liquid crystal phases replacing phase-separated (PS) regime. The three points are the same as in Fig. 3, defining the limits of stability of the surrounding phases. The lower panel shows symmetry-allowed 3d transition scenarios as a function of temperature to the normal-nematic $(\mathrm{N}-\mathrm{Nm})$, normal-isotropic $(\mathrm{N}-\mathrm{I})$, normal-smectic $\left(\mathrm{N}-\mathrm{Sm}_{2 Q}\right)$, and "charge"-4 superfluid-nematic $\left(\mathrm{SF}_{4}-\mathrm{Nm}\right)$ phases. Currently, no microscopic calculations exist that assess which of the three possible scenarios are actually realized in imbalanced Fermi gases and in what range of detuning.

$\theta_{s c}=\left(\theta_{+}+\theta_{-}\right) / 2$ and the domain-wall phonon, $u=\theta_{s m} / Q=\left(\theta_{+}-\theta_{-}\right) / 2 Q$, with the LO order parameter (in a single $\pm Q$ approximation) given by

$$
\Delta_{L O}(\mathbf{x})=2 \Delta_{Q} e^{i \theta_{s c}(\mathbf{x})} \cos \left(\mathbf{Q} \cdot \mathbf{x}+\theta_{s m}(\mathbf{x})\right) .
$$

The LO order parameter is a product of a superfluid order parameter and a unidirectional spontaneously oriented (along Q) Cooper-pair density wave, i.e., it simultaneously exhibits the off-diagonal long-range order and smectic order.

Symmetry arguments supported by detailed microscopic calculations [126] show that the low-energy modes of the LO state involve two coupled smectics (with the layer normal taken to lie along $\mathbf{x}_{\|}$), with moduli derivable from the BCS theory:

$$
\mathcal{H}_{L O}=\frac{K}{2}\left(\nabla^{2} u\right)^{2}+\frac{B}{2}\left(\partial_{\|} u+\frac{1}{2}(\nabla u)^{2}\right)^{2}+\frac{\rho_{s}^{i}}{2}\left(\nabla_{i} \theta_{s c}\right)^{2},
$$

a form that is familiar from studies of conventional smectic liquid crystals, with rotational invariance encoded through the vanishing of the $\left(\nabla_{\perp} u\right)^{2}$ modulus and the specific form of the nonlinear elastic terms. The LO state is found to be a highly 
anisotropic superfluid with the ratio of superfluid stiffnesses given by

$$
\rho_{s}^{\perp} / \rho_{s}^{\|}=\frac{3}{4}\left(\Delta_{Q} / \Delta_{\mathrm{BCS}}\right)^{2} \approx \ln \left(h_{c 2} / h\right) \ll 1,
$$

vanishing for $h \rightarrow h_{c 2}^{-}$. The FF state is found to be even more exotic, characterized by an identically vanishing transverse superfluid stiffness, a reflection of the rotational invariance of the spontaneous current to an energy-equivalent ground state.

Thus, a resonant imbalanced Fermi gas confined to an isotropic trap is a natural realization of a quantum (superfluid) liquid crystal that, unlike the solid state analogs, is not plagued by the underlying lattice potential that explicitly breaks continuous spatial symmetries.

It has been demonstrated [126] that in 3d the long-range LO order is stable to quantum fluctuations, but is marginally unstable at any nonzero $T$. The resulting superfluid state is an algebraic phase, characterized by universal quasi-Bragg peaks and correlations that admit an asymptotically exact description. In contrast, putative crystalline LO phases [127] with multiple noncolinear ordering wavevectors are stable against thermal fluctuations. The resulting state is also found[126] to exhibit an unusual topological excitation - a half vortex bound to a half dislocation - allowed by the above form of $\Delta(\mathbf{x})$ in Eq. (27).

In $2 \mathrm{~d}$, at nonzero $T$, the state is also an algebraic phase, exhibiting universal power-law phonon correlations, controlled by a nontrivial exactly calculable fixed point. However, it displays short-range positional order with Lorentzian structure-function peaks, and is thus unstable to the proliferation of dislocations. The resulting state is either a "charge"-4 (four-fermion condensate) superfluid (with order parameter $\Delta_{s c}^{(4)} \sim$ $\Delta^{2} \approx \frac{1}{2} \Delta_{Q}^{2} e^{i 2 \theta_{s c}}$, or a non-superfluid nematic, depending on the relative energetics of the aforementioned integer and half-integer vortex-dislocation defects. The latter normal nematic state is a (complementarily described [128]) deformed Fermi surface state [115, 116], that is another exotic candidate for a strongly interacting, imbalanced Fermi gas.

The unbinding of various combinations of topological defects (dislocations and vortices) yields predictions for a number of other interesting phases [126]. These include two types of aforementioned orientationally-ordered but statistically homogeneous (dislocations are unbound) nematic states, one where superfluidity is completely destroyed ("normal" nematic, N-Nm) and another in which 4-atom superfluid order accompanies the orientational nematic order ("superfluid" nematic, $\mathrm{SF}_{4}-\mathrm{Nm}$ ). Another state is the "normal" smectic phase, $\mathrm{N}-\mathrm{Sm}_{2 Q}$, emerging from the LO state by proliferation of vortices, that destroy the superconducting phase coherence, but retain the periodic density layering. The three possible symmetry-allowed scenarios for transitions between these liquid crystal phases as a function of temperature are illustrated in the lower panel of Fig. 6. Currently, no microscopic calculations exist that assess which of the three possible scenarios are actually realized in imbalanced Fermi gases and in what range of detuning.

A complete low-energy description of these LO liquid crystal states must take the 
gapless fermionic excitations into account, in addition to the above bosonic Goldstone modes. As with other analogous problems [115], these are expected to lead to the Landau-like damping of the Goldstone modes $\theta_{s c}, u$, and a finite fermionic quasi-particle lifetime. So far, this difficult problem has not been addressed.

\subsection{Experimental predictions}

The possibility of the observation of conventional FFLO states has been discussed at length [62, 129, 59, 66]. Bragg peaks (reflecting spontaneous periodicity) observed in time of flight [66] experiments and the spectra of collective breathing modes [130] are two prominent signatures. It has been recently emphasized that the FFLO states may be energetically stabilized in a quasi-1d geometry [131.

In the inhomogeneous environment of the trap there are a number of constraints on the observation of FFLO states and the fluctuation phenomena discussed above. Near $h_{c 2}$ the LO period $\lambda_{Q}=2 \pi / Q_{0}$ is bounded by the coherence length (that near unitarity can be as short as $\sim R / N^{1 / 3}$, where $R$ is the trapped condensate radius and $N$ is the total number of atoms), and thus $\ll R$ and in this regime the trap can be treated via a local density approximation (LDA). For $\lambda_{Q} \ll R$, LDA predicts a weak pinning of the LO smectic, that can be estimated via finite size scaling, with trap size $R$ cutting off $\left\langle u^{2}\right\rangle \sim \eta \log \left(R / \lambda_{Q}\right)$, leading to $\left\langle\Delta_{L O}\right\rangle \sim\left(\lambda_{Q} / R\right)^{\eta} \ll 1$ that no longer truly vanishes although it is still strongly suppressed. We expect the predicted strong-fluctuation effects to be experimentally accessible. We note, for example, that Kosterlitz-Thouless phase fluctuation physics has been reported in $2 \mathrm{~d}$ trapped superfluids [41], despite the finite trap size. However, a more detailed analysis of the trap effects, necessary near $h_{c 1}$ for a quantitative comparison with experiments, remains to be done.

Other experimental signatures of the LO state include vortex fractionalization, where the basic superfluid vortex is half the strength of a vortex in a regular paired condensate. This should be observable via a doubling of the vortex density in a rotated state. Also under rotation, the high superfluid anisotropy Eq. (29) is expected to lead to an imbalance-tunable strongly anisotropic vortex core and a lattice that is highly stretched along $\mathbf{Q}$. Bragg peaks in the time-of-flight images can distinguish the periodic $\mathrm{SF}_{2}-\mathrm{Sm}_{Q}$ (superfluid smectic) state from the homogeneous $\mathrm{SF}_{4}$-Nm (superfluid nematic), which are in turn distinguished from the $\mathrm{N}-\mathrm{Sm}_{2 Q}$ and $\mathrm{N}-\mathrm{Nm}$ (normal smectic and nematic) by their superfluid properties, periodicity, collective modes, quantized vortices, and condensate peaks. Thermodynamic signatures can identify the corresponding phase transitions.

\section{Local density approximation}

One of the principal experimental probes of ultra-cold atomic gases is the measurement of the local atom density of the trapped cloud. Thus, quite generally, detailed theoretical predictions for the phases and phenomena of cold polarized Fermi gases must account 

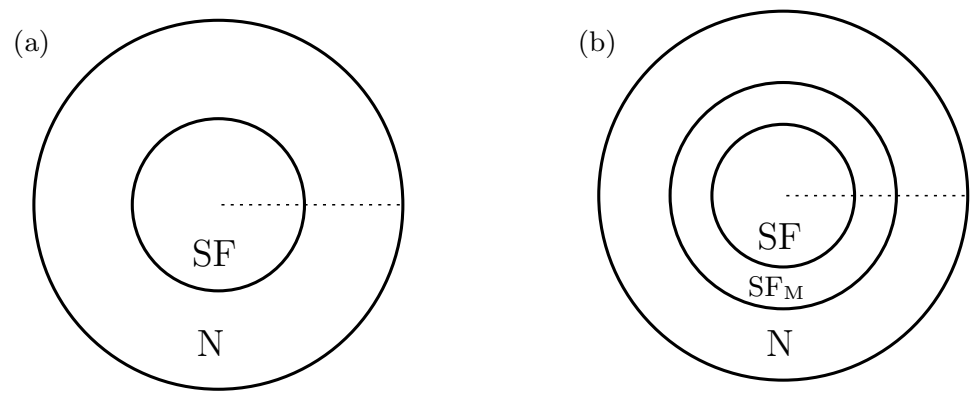

Figure 7. Schematic depiction of the shell structure of polarized spherical cold-atom clouds, with the phases occurring with increasing radius in a trapped polarized Fermi gas, within the LDA, (a) in the BCS and unitary regimes and in (b) the BEC regime where the $\mathrm{SF}_{\mathrm{M}}$ phase exists.

for the effect of the trapping potential $V_{T}(r)$. In recent years, there have been numerous theoretical investigations of the density profiles of trapped polarized fermion gases (see, e.g., Refs. 159, 132, 133, 134, 135, 136, 137, 120, 66, 138, 139, 140, 141, 142, 143); here we shall focus on the predictions of the simplest method for handling the trapping potential, namely the local density approximation (LDA).

Much like the WKB approximation, the LDA corresponds to using expressions for the bulk system, but with an effective local chemical potential $\mu(r)=\mu-V_{T}(r)$ in place of $\mu$. Systems for which the LDA holds, then, are of particular interest as they are relevant for the comparison to correlated condensed matter. The validity of the LDA relies on the smoothness of the trap potential, namely that $V_{T}(r)$ varies slowly on the scale of the longest physical length $\lambda$ (the Fermi wavelength, scattering length, effective range, etc.) in the problem, i.e., $\left(\lambda / V_{T}(r)\right) d V_{T}(r) / d r \ll 1$. Its accuracy can be equivalently controlled by a small parameter that is the ratio of the single particle trap level spacing $\delta E$ to the smallest characteristic energy $E_{c}$ of the studied phenomenon (e.g, the chemical potential, condensation energy, etc.), by requiring $\delta E / E_{c} \ll 1$.

Because of its more direct current experimental relevance, in this section we focus on the single-channel model, Eq. (17). The generalization of this model to a trap is straightforward:

$$
H=\sum_{\sigma=\uparrow, \downarrow} \int d^{3} r \hat{c}_{\sigma}^{\dagger}(\mathbf{r})\left[-\frac{\nabla^{2}}{2 m}+\left(V_{T}(\mathbf{r})-\mu_{\sigma}\right)\right] \hat{c}_{\sigma}(\mathbf{r})+\lambda \int d^{3} r \hat{c}_{\uparrow}^{\dagger} c_{\downarrow}^{\dagger} \hat{c}_{\downarrow} \hat{c}_{\uparrow},
$$

where $\hat{c}_{\sigma}(\mathbf{r})$ is a fermionic field operator with Fourier transform $\hat{c}_{\mathbf{k} \sigma}$. Henceforth, to be concrete, we shall focus on an isotropic harmonic trap $V_{T}(\mathbf{r})=V_{T}(r)=\frac{1}{2} m \Omega_{T}^{2} r^{2}$, although this simplification can be easily relaxed to handle an arbitrary anisotropic trap. The LDA assumes that, locally, the system is taken to be well approximated as uniform, but with a local chemical potential given by

$$
\mu(r) \equiv \mu-\frac{1}{2} m \Omega_{T}^{2} r^{2},
$$




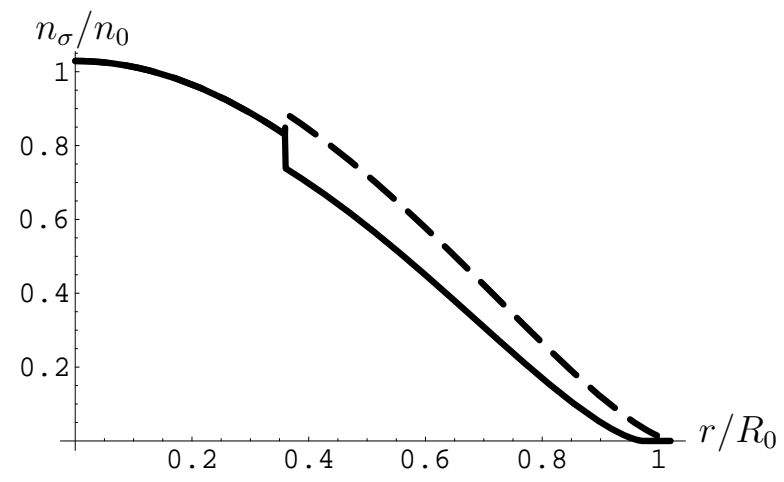

Figure 8. Plot of the local fermion densities, $n_{\uparrow}$ (dashed) and $n_{\downarrow}$ (solid), as a function of radius and normalized to the overall density $n_{0}$, as a function of position normalized to the $P=0$ Thomas-Fermi radius $R_{0}$, in the BCS regime with $\left(k_{\mathrm{F}}\left|a_{\mathrm{s}}\right|\right)^{-1}=1.5$, from Ref. 66].

where the constant $\mu$ is the true chemical potential (a Lagrange multiplier) still enforcing the total atom number $N$. The spatially-varying spin-up and spin-down local chemical potentials are then given by

$$
\begin{aligned}
& \mu_{\uparrow}(r)=\mu(r)+h, \\
& \mu_{\downarrow}(r)=\mu(r)-h,
\end{aligned}
$$

with the chemical potential difference $h$ uniform. Thus, within the LDA we approximate the energy density by that of a uniform system with spatial dependence (via the trap) entering only through $\mu(r)$. The ground state energy is then simply a volume integral of this energy density. Within LDA, the phase behavior as a function of chemical potential, $\mu$, translates into a spatial cloud profile through $\mu(r)$, with critical phase boundaries $\mu_{c}$ corresponding to critical radii defined by $\mu_{c}=\mu\left(r_{c}, h\right)$. As we first predicted [59], this leads to a shell-like cloud structure (illustrated in Fig. 7) that has subsequently been observed experimentally [55, 56, 57].

Below we briefly review these shell structures using the LDA, following our earlier work 66. We note, however, that throughout our discussion, sharp (discontinuous) features that arise are an artifact of LDA and are expected to be smoothed on microscopic (Fermi wave-) length scales by the kinetic energy (or, surface tension [137]).

We begin with the analysis in the BCS regime, where $\mu>0$. Qualitatively, the unitary regime will behave analogously to the BCS regime. Neglecting the FFLO phase, the fixed $\mu$ and $h$ phase diagram has a first-order phase transition to the normal phase at

$$
\begin{aligned}
& h_{c} \simeq \frac{1}{\sqrt{2}} \Delta_{\mathrm{BEC}-\mathrm{BCS}}(\mu), \\
& \simeq 4 \sqrt{2} \mathrm{e}^{-2} \mu(r) \exp \left[\frac{m}{4 \pi a_{\mathrm{s}} N(\mu(r))}\right],
\end{aligned}
$$

separating a polarized normal phase (when $h>h_{c}$ ) and a paired superfluid phase (when $h<h_{c}$ ). Since $\Delta_{\mathrm{BEC}-\mathrm{BCS}}(\mu)$ is an increasing function of $\mu$, this leads to the LDA 


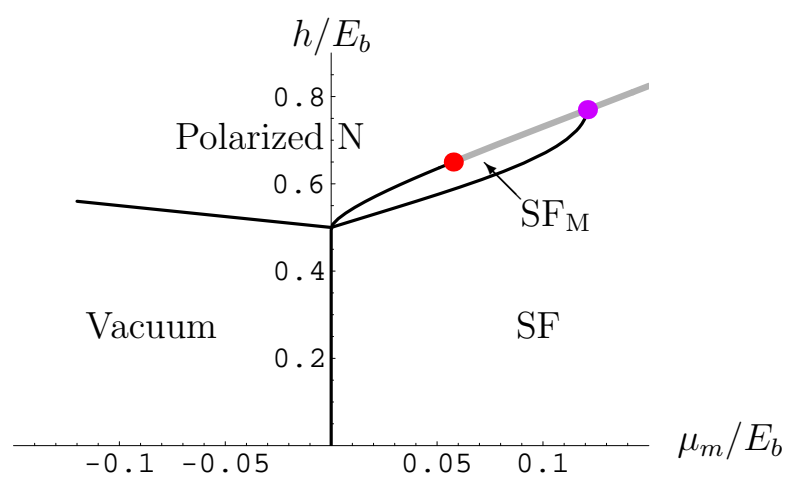

Figure 9. Phase diagram, in the grand-canonical ensemble at fixed $\mu$ and $h$, with $h$ normalized to the molecular binding energy $E_{b}=\frac{\hbar^{2}}{m a_{\mathrm{s}}^{2}}$ and $\mu_{m}=2 \mu+E_{b}$ the effective molecular chemical potential. The thick grey line is a first-order transition, and the thin black lines are continuous transitions. The red point is a tricritical point and the purple point is a critical end point.

prediction of a fully paired superfluid core at small $r$ (where $\mu$ is larger) surrounded by a population imbalanced normal phase, as illustrated in Fig. 7a. This yields a remarkably simple behavior for the spin- $\uparrow$ and spin- $\downarrow$ densities $n_{\uparrow, \downarrow}$, plotted in Fig. 8 , with $n_{\uparrow}=n_{\downarrow}$ in the central core (the fully-paired superfluid phase), and $n_{\uparrow} \neq n_{\downarrow}$ in the outer shell (the imbalanced unpaired Fermi liquid phase). We note here that including the FFLO phase, that occurs near the SF-N phase transition, would imply a thin shell of FFLO within the LDA yielding oscillations of the local pair amplitude near the SF-N interface, as also found in recent beyond-LDA analysis based on the BdG equations [133].

Turning to the BEC regime, Fig. 9 shows the homogeneous-case phase diagram at fixed $\mu$ and $h$ that is required to construct the phases occurring with increasing radius within the LDA. The structure of this phase diagram is somewhat nonintuitive; this is because at fixed densities the chemical potential in the BEC regime changes rapidly with external parameters (like the detuning), as also seen in the balanced case, see Fig. 1b. Nonetheless, the resulting shell structures are the natural generalization of the BCS regime results: Within the LDA, possible sequences of phases with increasing radius correspond to constant $h$ trajectories, with decreasing $\mu$, in the phase diagram of Fig. 9 , Thus, we see that the possible sequences of phases with increasing $r$ are: pure $\mathrm{SF}, \mathrm{SF} \rightarrow$ $\mathrm{N}$, or $\mathrm{SF} \rightarrow \mathrm{SF}_{\mathrm{M}} \rightarrow \mathrm{N}$, with the latter case illustrated in Fig. $7 \mathrm{~b}$.

The characteristic LDA profiles in the BEC regime for the local pair density $n_{m}(r)$ and magnetization $M(r)$ are shown in Fig. 10 for the sequence of phases $\mathrm{SF} \rightarrow \mathrm{SF}_{\mathrm{M}} \rightarrow \mathrm{N}$. Thus, as illustrated in Fig. 7 , in contrast to the BCS regime here the cloud can exhibit a thin shell of the $\mathrm{SF}_{\mathrm{M}}$ phase with finite polarization and a pairing gap. The three characteristic radii of importance are: $R_{f 1}$ (the $\mathrm{SF} \rightarrow \mathrm{SF}_{\mathrm{M}}$ transition), $R_{T F}$ (the ThomasFermi radius, where the $\mathrm{SF}_{\mathrm{M}} \rightarrow \mathrm{N}$ transition occurs) and $R_{f 2}$, the outer boundary of the cloud. As we discuss in the next section, the LDA has been used by the Ketterle group 108 to infer the phase diagram for a homogeneous system. However, interesting physics also holds for systems in which LDA is violated. As discussed 


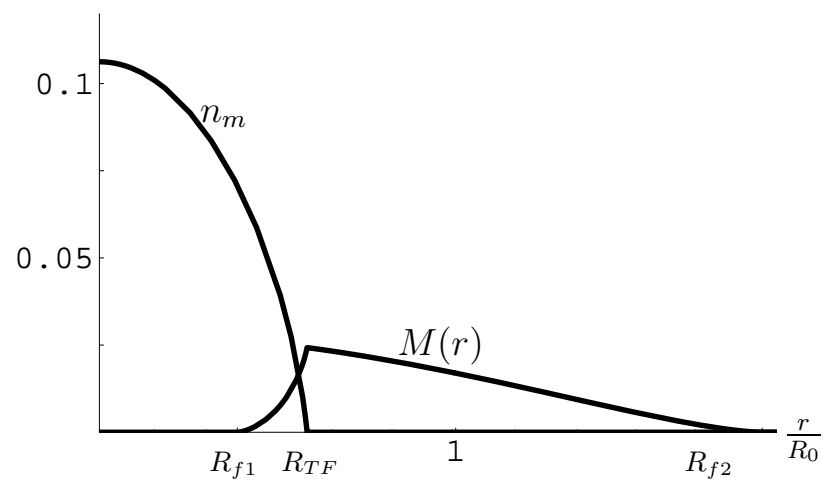

Figure 10. Typical pair density, $n_{m} \propto|\Delta|^{2}$, and local magnetization, $M$, each normalized to a characteristic density scale $E_{b} N\left(E_{b}\right)$, with $N\left(E_{b}\right)$ the density of states measured at the binding energy, from Ref. [66] in the BEC regime.

by DeSilva and Mueller [135], the early Rice experiments [56] showed axial density profiles (corresponding to the density, integrated over two directions, as a function of the coordinate $z$ along the long axis of the cloud) that apparently violated the LDA. Indeed, the violation of the LDA can be directly seen in the data shown in Fig. 1 of Ref. [56], since the fermion densities do not follow the contours of the large aspect ratio trap. Additionally DeSilva and Mueller showed (see also Ref. [136]) that for LDA-type cloud shapes of the form of Fig. 7, the axial magnetization must decrease monotonically with increasing $z$. A principal culprit in the breakdown of LDA is the surface tension associated with the SF-N interface [137]. This issue was studied in detail in Ref. [143] in an attempt to understand the density profiles from the Rice group [56, 58]. However, it was found theoretically that although surface tension indeed deforms the minority cloud in a way qualitatively consistent with the experiments, the size of the deformation is too small to account for experimental observations based on microscopic estimates of surface tension magnitude. This leaves a quantitative understanding of the Rice experiments an open question; one possible important ingredient that needs to be included are fluctuations around mean-field theory (known to be important in the unitary regime), as discussed in recent work [142].

\section{Recent experiments}

We now turn to a more comprehensive discussion of recent experimental developments in imbalanced Feshbach-resonant fermionic atomic gases. These have primarily been led by the Ketterle group at MIT and the Hulet group at Rice, launched by two studies of ${ }^{6} \mathrm{Li}$ that appeared in Science in 2006 [55, 56].

The MIT experiments [55] reported a destruction of superfluidity (probed by the 
appearence of vortices in response to an imposed rotation) and condensation (probed by a fit to the cloud shape after sweeping to the BEC side of the resonance) with increasing imbalance. As shown in Fig. 1 of Ref. [55], a rotating polarized Fermi gas exhibits a distinctive inner superfluid core (defined by a region to which the induced vortex lattice is confined) surrounded by a vortex-free shell of imbalanced normal cloud. Consistent with this identification, Fig. 2 of Ref. [55] shows that the number of vortices $N_{v}$ decreases with increasing imbalance for different values of the detuning. On the basis of the standard relation [144] $N_{v}=2 m \Omega A / \pi \hbar$, we thus observe that $N_{v}$ simply measures the cross-sectional area $A$ (normal to the axis of rotation) of the superfluid core as a function of the rotation rate $\Omega$. Although qualitatively this data (the shrinking of the superfluid core with increasing imbalance) is consistent with current theoretical predictions, these observations remain to be quantitatively understood. Zwierlein, et al. [55] also reported evidence of phase separation, as reflected in a suppression of the local polarization (species imbalance) near the center of the cloud, a signature of a core that is fully-paired into a BEC-BCS singlet superfluid. These were confirmed by in situ measurements of individual species density profiles using an exquisite phase-contrast imaging and 3d image reconstruction technique[57]. Based on these observations they constructed a phase diagram [55] with a phase boundary separating the normal phase and superfluid phases (the latter identified with normal-superfluid phase separation), in qualitative agreement with the mean-field theoretical phase diagram [59, 66].

The initial Rice experiments [56] studied the imbalanced ${ }^{6} \mathrm{Li}$ Fermi gas by comparing the density profiles of the two fermion species. Consistent with the MIT experiments they found that, with increasing species number imbalance, the imposed imbalance is pushed to the outside shell of the cloud. Furthermore, by measuring the dependence of the radii of the Thomas-Fermi clouds for the two fermion species and showing that the ratio of the majority to minority radius, $R_{\uparrow} / R_{\downarrow}$, increases with increasing imbalance $P$, Partridge, et al. showed evidence for phase separation in the unitary regime. From these measurements, the Rice group found a putative nonzero lower-critical polarization for phase separation with $P_{c 1} \simeq 0.09$, observing that for $P<P_{c 1}$ the radii of the two species coincided, $R_{\uparrow}=R_{\downarrow}$, despite a nonzero imposed imbalance (see Fig. 3 of Ref. [56]). We are not aware of a ground state (within a comprehensive theoretical picture) that can accomodate this observation of a nonzero $P_{c 1}$, i.e., a state that is both fully paired and imbalanced; naive candidates like the homogeneous polarized superfluid $\mathrm{SF}_{M}$ (appearing on the BEC side) [59, 66] and a putative $p$-wave paired state [145] would fail toward the edge of the cloud, where on general grounds a polarized normal state with distinct radii is expected. Furthermore, a reasonable understanding of this data was obtained by Chevy [81] within a simple unitary model of phase separation (i.e., with distinct majority-minority radii) between a fully-paired unpolarized superfluid and a fully-polarized normal state, using universal forms for the equations of state for these phases. Taking these together with the fact that no such finite $P_{c 1}$ was ever observed by the MIT group [57] suggests that the putative (small) nonzero $P_{c 1}$ in Rice measurements may be an experimental artifact. An alternative possibility is that it is a consequence 
of a nonzero temperature $T \approx 0.1 T_{F}$, that on one hand allows a finite polarization (through thermally activated quasi-particles) and on the other entropically suppresses phase separation, thereby allowing a nonzero $P_{c 1}$. One support for the latter scenario is the fact that in the later Rice experiments [58] a vanishing $P_{c 1}$ was found at reduced temperature, $T \approx 0.05 T_{F}$. Detailed experiments further clarifying this issue would be highly desirable.

A number of subsequent experiments further explored the onset of phase separation in such imbalanced Fermi gases. Zwierlein, et al. [55] studied the transition between the unpaired and superfluid phases by observing column density profiles in imbalanced Fermi gases, finding the upper-critical polarization of $P_{c 2 \text {,trap }} \simeq 0.70$ at the unitary point for the trapped cloud. Due to the presence of the trap, this value cannot be directly compared to the upper-critical polarization $P_{c 2}$ for the bulk, uniform system, the latter estimated within mean-field analysis to be $P_{c 2}^{\mathrm{mft}} \simeq 0.93$. However, within the LDA, quite generally

$$
P_{c 2, \text { trap }}>P_{c 2} \text {. }
$$

This bound that can be understood by considering a trapped imbalanced Fermi gas at $P_{c 2 \text {,trap }}=M / n=\Delta N / N$, with magnetization $M=\Delta N / V$ ( $V$ the system's volume) and atom density $n=N / V$. Within the LDA, at $P_{c 2 \text {,trap }}$, by definition the fully-paired inner superfluid core has shrunk to zero radius. Thus, at the center of the trap the local polarization is given by the bulk upper-critical polarization, i.e., $P(r=0)=M(0) / n(0)=P_{c 2}$, where $M(r)$ is the local magnetization and $n(r)$ is the local density. Now, since $\mu(r)=\mu-\frac{1}{2} m \Omega^{2} r^{2}$ decreases with increasing radius, and the chemical potential difference $h$ is $r$-independent, $M(r)$ and $n(r)$ respectively increase and decrease with increasing radius, implying

$$
\begin{aligned}
\Delta N & =\int d^{3} r M(r) \geq V M(0), \\
N & =\int d^{3} r n(r)<V n(0) .
\end{aligned}
$$

and giving the bound in Eq. (36). Thus, the observation of $P_{c 2 \text {,trap }} \simeq 0.70$ in Ref. [55] is a measure of a lower-bound on the inaccuracy of the mean-field estimate for $P_{c 2}$.

The imaging technique in such experiments naturally integrates the local atomic density along the axis perpendicular to the camera's imaging plane. To deconvolve this projection, Shin, et al. [57] used the inverse Abel transform [22] to extract the local three-dimensional atom densities. This development allowed a clear identification of the paired superfluid core (within which the local imbalance identically vanishes, at $T=0$ ) in the phase separation regime, and the measure of the upper-critical polarization as a function of detuning, finding $P_{c 2 \text {,trap }}=0.77$ at unitarity, close to unity in the BEC regime, and decreasing in the BCS regime.

One of the most significant differences between the MIT and Rice observations is the shape of the interface between the balanced superfluid core and the imbalanced outside shell. While the MIT group found an interface whose shape mimics the trap 
(see, e.g., Fig. 6 of Ref. [57]), the Rice group observed that the imposed imbalance is accomodated by an enhanced local polarization in the axial poles of the cloud [56], thereby at low $T$ deviating from the trap's highly anisotropic shape. This discrepancy, which to date remains to be quantitatively understood, is believed to be related to the very different trap aspect ratios in the $\operatorname{MIT}\left(f_{r} / f_{z}=5.6\right.$ in Ref. [57]) and Rice $\left(f_{r} / f_{z}=49\right.$ in Ref. [56]) experiments, the latter exhibiting a nearly one-dimensional trap geometry. This issue was studied in more detail in Ref. [58], where the distorted shape (attributable to a nonzero surface tension [135] associated with the first-order transition and corresponding phase separation) of the paired inner core was confirmed, and the dependence of the aspect ratios of the minority and majority species fermionic clouds as a function of the imposed imbalance was measured. These later Rice experiments [58] also found a surprisingly large value of the in-trap upper critical polarization, $P_{c 2 \text {,trap }} \approx 0.9$. Subsequent Rice experiments [146] explored the effects of trap aspect ratio, and found that the LDA-violating deformations decrease with decreasing aspect ratio, with no deformations observed for aspect ratios below 6. Furthermore, the critical polarization was found to be approximately $P \approx 0.75$, consistent with the MIT measurements and Monte Carlo calculations.

However, recent Paris experiments [74] studied the phase diagram of an imbalanced Fermi gas with around $10^{5}$ atoms and trap aspect ratio of around 20, conditions in between the Rice and MIT systems. In contrast to the above appealing resolution of the discrepancy between the Rice and MIT observations (due to a very different aspect ratio), these Paris experiments saw no appearance of LDA-violating features or surface tension effects. Furthermore their results are in a very good agreement with MIT's, both qualitatively (observing a flat-top distribution of the density difference, that can arise from a fully-paired superfluid core within the LDA), and quantitatively (with respect to the critical polarization $P_{c 2}$ ). Thus, a resolution of these puzzling discrepancies remains an open issue.

The observed matching of the majority and minority clouds' aspect ratios to the trap's aspect ratio in the MIT experiments indicates that, for these experiments, the LDA is well satisfied. The MIT group then took advantage of the LDA to extract the $T=0$ and finite $T$ phase diagrams [108, 109] for a bulk system, facilitating a more direct comparison with theoretical studies of a homogeneous gas. Focusing on the unitary limit, these experiments found a finite- $T$ phase diagram of the standard tricritical form (i.e., like Fig. 5, resembling other tricritical systems, such as ${ }^{3} \mathrm{He}-{ }^{4} \mathrm{He}$ mixtures [104], metamagnets [147], and thin-film superconductors [148]).

As illustrated in Fig. 5, a tricritical point separates a second-order (at high $T$ ) from a first-order (at low $T$ ) transition that, in the present case, is between a paired superfluid and a polarized normal state, with the latter extending to a regime of phase separation at fixed polarization. This general structure is well captured by mean-field theory for the narrow-resonance model [68], however the shape of the phase diagram in the experimentally-relevant wide-resonance regime remains an open question. Finite temperature analysis beyond mean-field theory [149] finds a more complicated phase 
diagram topology, with the regime of phase separation persisting for temperatures higher than the tricritical point.

The principal experimental evidence for the expected tricritical point comes from the observations [58, 108] that at higher temperatures the breakdown of LDA (as signaled by the distinct cloud and trap shape anisotropies) ceases. This was reasonably interpreted as the disappearance of the surface tension (associated with phase coexistence at first-order transition) as the low- $T$ first-order superfluid-normal transition converts to a continuous one for $T$ above the tricritical temperature. This is further supported by observations that at these higher temperatures (above the tricritical temperature) the expected Clogston limit of $P_{c 2 \text {,trap }} \approx 0.75$ is recovered. A more detailed check on the consistency of the interpretation can come from a comparison with the predictions for the cloud shape, heat capacity, and local polarization controlled by the mean-field tricritical point [117], which are predicted to follow distinct power laws [150, 151].

As mentioned above, in later work [109], Shin, et al. also used the LDA to extract the $T=0$ phase diagram of an imbalanced Fermi gas. The resulting experimental phase diagram agrees qualitatively with the theoretical one displayed in Fig. 3] [59, 66], though no experimental evidence for the putative FFLO phase exists to date, nor has the low- $T$ $\mathrm{SF}_{\mathrm{M}}$-to-PS phase boundary $\left(P_{\mathrm{c} 1}\right)$ been mapped out. The observed value of $P_{c 2} \simeq 0.36$ at unitarity (reported in Ref. [108, with the phase diagram of Ref. [109] showing a slightly lower value) is consistent with the above bound $P_{c 2}<P_{c 2 \text {,trap }}$ and points to a large quantitative error in the mean-field estimate (although including the leading $1 / N_{f}$ correction [83] yields a more accurate estimate, $P_{\mathrm{c} 2} \simeq 0.302$ ) This measurement is consistent with the quantum Monte Carlo based prediction by Lobo, et al [112], who find $n_{\uparrow} / n_{\downarrow}=0.44$ at the transition, corresponding to $P_{c 2}=0.38$ and within LDA giving a universal value of $P_{c 2 \text {,trap }}=0.77$, in agreement with the MIT [57] and latest Rice [146] measurements.

One remarkable aspect of the experiments in Ref. [109] is that the density profiles of the trapped imbalanced Fermi gases were quantitatively captured within a simple bosonfermion mixture model (with bosons representing the tightly bound molecular pairs and fermions representing the excess spin-up fermions), using the vacuum moleculefermion [152] $\left(a_{b f}=1.18 a\right)$ and molecule-molecule [153, 85] $\left(a_{b b}=0.6 a\right)$ scattering lengths. Since the latter values are only valid in the dilute deep-BEC regime, it is surprising that such an accurate fit to the data held close to unitarity. It will be interesting, in future work, to test the limits of the boson-fermion model used in Ref. [109] and to look for deviations from this simple picture.

Before turning to the last topic of this section, which is the development of RF spectroscopy probes of imbalanced Feshbach-resonant Fermi gases and the stronglyimbalanced polaron regime, we briefly comment on recent Rice experiments [154], that have attained the quasi one-dimensional conditions for which the 1d FFLO state is expected. These experiments utilize a two-dimensional optical lattice to create an array of weakly-coupled, large aspect ratio tubes (with $\omega_{r} / \omega_{z}=1000$ ), that are 
therefore effectively in a one-dimensional limit. Previous theoretical work [131] and general arguments suggest that in this limit the FFLO state should robustly appear. Moreover, uniform one-dimensional imbalanced Fermi gases can be solved exactly via Bethe ansatz [155, 156], supporting these claims. Liao et al [154] found that, combining such Bethe ansatz results with LDA (to account for the spatial dependence along the tube axis), a quantitative agreement with their trapped density profiles was obtained. Consistent with earlier theoretical predictions [131], these experiments found that in contrast to the $3 \mathrm{~d}$ case, the superfluidity is weakest and the imbalance (magnetization) is largest at the center of the trap where the chemical potential is smaller. This is a consequence of the reduction in the $1 \mathrm{~d}$ density of states with the increasing chemical potential (contrasting with the opposite behavior in $3 \mathrm{~d}$ ), resulting in the appearance of the balanced superfluid at small $\mu$, and the imbalanced superfluid (the $1 \mathrm{~d}$ equivalent of the FFLO state) at large $\mu$ at the center of the trap. An important goal for future work is a direct observation of the expected modulation of the FFLO state. This should be reflected in quantities such as the local pairing correlations, as found in recent QMC [157] and density-matrix renormalization group [158, 159] studies of one-dimensional systems. Finite momentum Bragg BEC peaks (akin to those appearing in a condensates in an optical lattice, but here without an imposed periodic potential) are also expected to characterize the time-of-flight measurements of the FFLO states.

We now discuss RF spectroscopy [17], which has been fruitfully used to probe resonant imbalanced Fermi gases, with the particular focus on the strongly interacting normal state driven by a high imbalance and/or increased temperature [72, 160]. RF spectroscopy studies of strongly imbalanced Fermi gases have a close condensed-matter counterpart, namely tunneling in the paramagnetic phase above the Clogston limit in thin-film superconductors [161, 162. Instead of removing or adding electrons through an insulating barrier, in RF spectroscopy the RF field induces transitions from one of the two interacting hyperfine species of the atomic cloud to a third unpopulated hyperfine state, and this rate as a function of the RF frequency $\omega$ is measured by detecting the number $N_{3}(\omega)$ of the third species. In the simplest interpretation the signal $N_{3}(\omega)$ measures the spectral function of the species undergoing a transition to the third hyperfine state.

However, as was recently emphasized [90, 163, 164, 165, 166, 167, 168], this interpretation is complicated by final state interactions (a crucial issue that has received much recent theoretical attention [90, 163, 164, 165, 166, 167, 168]) of the third hyperfine state that are strong in ${ }^{6} \mathrm{Li}[72$, 160], and a significantly more involved theoretical analysis is required. (In contrast, in ${ }^{40} \mathrm{~K}$ final state interactions are weak and a direct comparison with the fermionic spectral function is possible [169]).

The key features of the RF signal are the width and the shift of the peak in $N_{3}(\omega)$ relative to that in a cloud of noninteracting atoms. Based on earlier studies[17] the observed peak shift was originally associated with the pairing gap. An observation of a such shift for a strongly imbalanced gas in the regime $\left(T, P, k_{F} a\right)$ of the phase diagram, where the gas did not display a condensate peak, was taken as evidence for an exotic 
paired non-superfluid state[72]. This is reminiscent of photoemission in the cuprate high-temperature superconductors, which exhibit a pseudogap [45], loosely taken as evidence of local pairing correlations [170, 171, 172, 38] in the non-superconducting state above $T_{c}$. However, a detailed analysis [90] (including final state interactions and controlled by a systematic $1 / N_{f}$-expansion [82, 83]) of the imbalanced resonant gas demonstrated that the observed RF shift and widths can be qualitatively understood in terms of a conventional Fermi-liquid state, albeit a strongly renormalized one. The latter is characterized by large self-energy corrections leading to a modified Migdal discontinuity in the momentum distribution function (single-particle residue), $Z<1$, the enhanced effective mass, $m^{*}>m$, and a large chemical potential shift responsible for the observed shift in the RF peak[90]. Subsequent experiments from the same group[160]

have successfully reinterpreted the original observations in terms of this theoretically advocated Fermi-liquid picture, though a detailed quantitative description of the data is still lacking.

Finally, as mentioned earlier recent experimental studies [73, 74] have provided additional insight on this interesting system in the strongly imbalanced limit of $P \rightarrow 1$, corresponding to a single minority (e.g., spin down) atom moving and resonantly interacting with a fully polarized Fermi sea of majority atoms (spin up). Schirotzek, et al [73] find evidence for the predicted transition [173, 113] from a polaron-like state of the minority atom interacting with the majority Fermi sea, characterized by a nonzero fermionic pole with a residue $Z<1$, to a diatomic bosonic molecule in the Fermi sea, with $Z \rightarrow 0$. Subsequent work by Nascimbene, et al. [74] studied the collective oscillations of the strongly imbalanced Fermi gas, extracting the effective polaron mass $m^{*} \simeq 1.17 m$. These measurements are in good agreement with simple variational calculation based on the Chevy ansatz [81, 111] and diagrammatic Monte-Carlo[173], that both give $m^{*}=1.17 m$, and another recent calculation [111] that yields $m^{*}=1.20 \mathrm{~m}$.

\section{Concluding remarks and future outlook}

In this brief review, we have discussed recent developments in the study of imbalanced resonant Fermi gases. As we described, their phenomenology is summarized by the phase diagram as a function of imbalance (polarization $P$ ) and interaction $\left(1 / k_{F} a\right.$, controlled by FR detuning). The zero-polarization paired-superfluid ground state, exhibiting the well-studied BEC-BCS crossover, is readily destablized by an imposition of a finite species imbalance, on the BCS side, to a regime of phase separation between the paired superfluid and a polarized normal state. In contrast, sufficiently deep in the BEC regime side this is preempted by a polarized homogeneous superfluid, consisting of molecular Bose-condensed pairs and a fully polarized Fermi sea of the remaining majority-species atoms. Beyond the so-called Clogston limit of the upper-critical imbalance, the strongly interacting normal state is obtained. The properties of these phases and regimes have been vigorously studied through measurements of the number and imbalance density profiles, collective modes, as well as the RF spectroscopy as a function of interaction 
strength (controlled by FR detuning), imbalance and temperature, finding qualitative and at times quantitative agreement with theoretical predictions.

Despite this coherent picture that has emerged, there are number of interesting issues that remain to be explored. Firstly, more detailed and quantitative studies of the phase boundaries are needed, in particular a determination of the $\mathrm{SF}_{M}$ phase boundary $P_{c 1}$ and the upper and lower critical polarizations as a function of detuning and temperature. In particular, it is not known whether the finite- $T$ phase diagram has the standard tricritical form for all detunings.

Secondly, more detailed investigations of phases are needed. As discussed in the manuscript, the polarized superfluid $\mathrm{SF}_{M}$, appearing in the $\mathrm{BEC}$ regime at $T=0$, is described, at the simplest level, by a simple Bose-Fermi mixture of molecular pairs and single-species Fermi sea. It would be interesting to explore further the extent to which this picture is only an approximation and what the limit of its validity is, particularly as the system is taken closer to the unitary point, where $s$-wave scattering length grows and the molecules can no longer be treated as point bosons.

There is also much that remains to be elucidated about the nature of the strongly interacting normal state at high polarization. Undoubtedly, RF spectroscopy and its recently-developed momentum-resolved extension [169] will be central to detailing its properties as a function of detuning and temperature.

But probably the biggest remaining enigma is the long sought-after FFLO state, or more generally an imbalance-driven paired superfluid state that spontaneously breaks translational and orientational symmetries. On the theoretical side, the form of the periodic state (unidirectional or crystalline and of what type [127]), the range of stability of the state in the detuning-polarization-temperature phase diagram, as well as the nature of the transition to it from the paired BEC-BCS superfluid remain as important open questions. Furthermore, as recently demonstrated the simplest LO state admits fractional topological defects (a 1/2-vortex bound to a 1/2-dislocation), and exhibits enhanced fluctuations that lead to a variety of other quantum liquid crystal phases and other rich phenomena[126].

As discussed above, reliable analytical treatments around $h_{c 2}$ 97 and those specializing to the FF (single $Q$ ) state 96, and more recent ones that extend these beyond the deep BCS regime[59, 66] suggest that the FFLO-type states are confined to a narrow sliver near $h_{c 2}$, just below the normal state. Whether this is generic or an artifact of current approximations remains an open question.

One of the reliable limits that sheds some light on this question is the case of one dimension, where there is an exact solution of the Bogoliubov-deGennes equations, predicting a LO type ground state over a large portion of the phase diagram 120 , 155, 156. This one-dimensional regime is also particularly amenable to numerical treatments [157, 158, 159, 174, 175] and exact Bethe-ansatz methods [155, 156]. In fact in one dimension, on general grounds the LO type state is generic for a finite imbalance, indistinguishable from the $1 \mathrm{~d} \mathrm{SF}_{M}$ ground state. Thus, the existence of a FFLO type state in one dimension is theoretically indisputable. Stimulated by this and supported 
by quasi-1d mean-field analysis[131], recent efforts have focused on a realization of the FFLO state in an array of weakly coupled 1d imbalanced resonant Fermi gases, created by a strong $2 \mathrm{~d}$ optical standing wave potential[146]. In the time of flight, this state is expected to exhibit spontaneous Bragg peaks associated with the simultaneous presence of superfluid coherence and periodic positional order. Undoubtedly, many unanticipated surprises will emerge as a result of these and many other future studies.

Acknowledgements We gratefully acknowledge past collaborations with V. Gurarie, A. Lamacraft, E.G. Moon, S. Sachdev, and M. Veillette, on which parts of this review are based, and stimulating discussions with D. Jin, E. Cornell, R. Hulet, and N. Navon, as well as financial support from NSF DMR-0321848 and the Louisiana Board of Regents, under grant No. LEQSF (2008-11)-RD-A-10.

\section{References}

[1] M.H. Anderson, J.R. Ensher, M.R. Matthews, C.E. Wieman, and E.A. Cornell, Science 269, 198 (1995).

[2] K.B. Davis, M.-O. Mewes, M.R. Andrews, N.J. van Druten, D.S. Durfee, D.M. Kurn, and W. Ketterle, Phys. Rev. Lett. 75, 3969 (1995).

[3] M.R. Matthews, B.P. Anderson, P.C. Haljan, D.S. Hall, C.E. Wieman, and E.A. Cornell, Phys. Rev. Lett. 83, 2498 (1999).

[4] K.W. Madison, F. Chevy, V. Bretin, and J. Dalibard, Phys. Rev. Lett. 86, 4443 (2001).

[5] J.R. Abo-Shaeer, C. Raman, J.M. Vogels, and W. Ketterle, Science 292, 476 (2001).

[6] I. Coddington, P.C. Haljan, P. Engels, V. Schweikhard, S. Tung, Phys. Rev. A 70, 063607 (2004).

[7] M. Greiner, O. Mandel, T. Esslinger, T.W. Hansch, and I. Bloch, Nature 415, 39 (2002).

[8] M.P.A. Fisher, P.B. Weichman, G. Grinstein, and D.S. Fisher, Phys. Rev. B 40, 546 (1989).

[9] D. Jaksch, C. Bruder, J.I. Cirac, C.W. Gardiner, and P. Zoller, Phys. Rev. Lett. 81, 3108 (1998).

[10] B. DeMarco and D.S. Jin, Science 285, 1703 (1999).

[11] C.A. Regal, M. Greiner, and D.S. Jin, Phys. Rev. Lett. 92, 040403 (2004).

[12] M.W. Zwierlein, C.A. Stan, C.H. Schunck, S.M.F. Raupach, A.J. Kerman, and W. Ketterle, Phys. Rev. Lett. 92, 120403 (2004).

[13] J. Kinast, S.L. Hemmer, M.E. Gehm, A. Turlapov, and J.E. Thomas, Phys. Rev. Lett. 92, 150402 (2004).

[14] M. Bartenstein, A. Altmeyer, S. Riedl, S. Jochim, C. Chin, J.H. Denschlag, and R. Grimm, Phys. Rev. Lett. 92, 203201 (2004).

[15] T. Bourdel, L. Khaykovich, J. Cubizolles, J. Zhang, F. Chevy, M. Teichmann, L. Tarruell, S.J.J.M.F. Kokkelmans, and C. Salomon, Phys. Rev. Lett. 93, 050401 (2004).

[16] G.B. Partridge, K.E. Strecker, R.I. Kamar, M.W. Jack, and R.G. Hulet, Phys. Rev. Lett. 95, 020404 (2005).

[17] C. Chin, M. Bartenstein A. Altmeyer, S. Riedl, S. Jochim, J.H. Denschlag, and R. Grimm, Science 305, 1128 (2004).

[18] M.W. Zwierlein, J.R. Abo-Shaeer, A. Schirotzek, C.H. Schunck, and W. Ketterle, Nature 435, 1047 (2005).

[19] I. Bloch, J. Dalibard, W. Zwerger, Rev. Mod. Phys. 80, 885 (2008).

[20] V. Gurarie and L. Radzihovsky, Annals of Physics 322, 2 (2007).

[21] S. Giorgini, L.P. Pitaevskii, and S. Stringari, Rev. Mod. Phys. 80, 1215 (2008).

$[22]$ W. Ketterle and M. Zwierlein, "Making, probing and understanding ultracold Fermi gases", in Ultracold Fermi Gases, Proceedings of the International School of Physics "Enrico Fermi", Course CLXIV, Varenna, 20 - 30 June 2006, edited by M. Inguscio, W. Ketterle, and C. Salomon.

[23] E. Timmermans, P. Tommasini, M. Hussein, A. Kerman, Phys. Rep. 315, 199 (1999). 
[24] E.A. Donley, N.R. Claussen, S.L. Cornish, J.L. Roberts, E.A. Cornell and C.E. Wieman, Nature 412, 295 (2001).

[25] C.A. Regal and D.S. Jin, Phys. Rev. Lett. 90, 230404 (2003).

[26] M. Bartenstein, A. Altmeyer, S. Riedl, R. Geursen, S. Jochim, C. Chin, J.H. Denschlag, R. Grimm, A. Simoni, E. Tiesinga, C.J. Williams, and P.S. Julienne, Phys. Rev. Lett. 94103201 (2005).

[27] A.V. Andreev, V. Gurarie, and L. Radzihovsky, Phys. Rev. Lett. 93, 130402 (2004).

[28] R.A. Barankov and L.S. Levitov, Phys. Rev. Lett. 93, 130403 (2004).

[29] E. Altman and A. Vishwanath, Phys. Rev. Lett. 95, 110404 (2005).

[30] N. Strohmaier, Y. Takasu, K. Gunter, R. Joerdens, M. Kohl, H. Moritz, and T. Esslinger, Phys. Rev. Lett. 99, 220601 (2007).

[31] A. J. Leggett, in Modern Trends in the Theory of Condended Matter, edited by A. Pekalski and J. Przystawa, Lecture Notes in Physics, Vol. 115 (Springer-Verlag, Berlin, 1980), p. 13.

[32] P. Nozières and S. Schmitt-Rink, J. Low Temp. Phys. 59, 195 (1985).

[33] C.A.R. Sá de Melo, M. Randeria, and J.R. Engelbrecht, Phys. Rev. Lett. 71, 3202 (1993).

[34] E. Timmermans, K. Furuya, P.W. Milonni, and A.K. Kerman, Phys. Lett. A 285, 228 (2001).

[35] M. Holland, S.J.J.M.F. Kokkelmans, M.L. Chiofalo, and R. Walser, Phys. Rev. Lett. 87, 120406 (2001).

[36] Y. Ohashi and A. Griffin, Phys. Rev. Lett. 89, 130402 (2002); Phys. Rev. A 67, 033603 (2003).

[37] J. Stajic, J.N. Milstein, Q. Chen, M.L. Chiofalo, M.J. Holland, and K. Levin, Phys. Rev. A 69, 063610 (2004).

[38] Q. Chen, J. Stajic, S. Tan, and K. Levin, Phys. Rep. 412, 1 (2005).

[39] Z. Nussinov and S. Nussinov, Phys. Rev. A 74, 053622 (2006).

[40] B. Paredes, A. Widera, V. Murg, O. Mandel, S. Fölling, I. Cirac, G.V. Shlyapnikov, T. Hänsch, and I. Bloch, Nature 429, 277 (2004).

[41] Z. Hadzibabic, P. Kruger, M. Cheneau, B. Battelier, and J.B. Dalibard, Nature 441, 1118 (2006).

[42] In the literature a variety of notations are used to describe the imposed population imbalance, including the most physical hyperfine species population imbalance (or simply imbalance), polarization and magnetization. We will use these terms interchangeably.

[43] R. Casalbuoni and G. Nardulli, Rev. Mod. Phys. 76, 263 (2004).

[44] M.G. Alford, K. Rajagopal, T. Schaefer, and A. Schmitt, Rev. Mod. Phys. 80, 1455 (2008).

[45] T. Timusk and B.W. Statt, Rep. Prog. Phys. 62, 61 (1999).

[46] C.M. Varma, P.B. Littlewood, S. Schmitt-Rink, E. Abrahams, and A.E. Ruckenstein, Phys. Rev. Lett. 63, 1996 (1989).

[47] G.R. Stewart, Rev. Mod. Phys. 73, 797 (2001).

[48] P. Coleman, in Lectures on the Physics of Highly Correlated Electron Systems VI, F. Mancini, Ed., American Institute of Physics, New York (2002), p. 79 - 160.

[49] S. Sachdev, Quantum Phase Transitions, Cambridge University Press, Cambridge, 1999.

[50] A.M. Clogston, Phys. Rev. Lett. 9, 266 (1962).

[51] G. Sarma, J. Phys. Chem. Solids 24, 1029 (1963).

[52] T.-L. Ho, Phys. Rev. Lett. 87, 060403 (2001).

[53] M.Y. Veillette, D.E. Sheehy, L. Radzihovsky, and V. Gurarie, Phys. Rev. Lett. 97, 250401 (2006).

[54] Y.-P. Shim, R.A. Duine, and A.H. MacDonald, Phys. Rev. A 74, 053602 (2006).

[55] M.W. Zwierlein, A. Schirotzek, C.H. Schunck, and W. Ketterle, Science 311, 492 (2006).

[56] G.B. Partridge, W. Li, R.I. Kamar, Y.-A. Liao, R.G. Hulet, Science 311, 503 (2006).

[57] Y. Shin, M. W. Zwierlein, C. H. Schunck, A. Schirotzek, W. Ketterle, Phys. Rev. Lett. 97, 030401 (2006).

[58] G.B. Partridge, W. Li, Y. A. Liao, R. G. Hulet, M. Haque, and H.T.C. Stoof, Phys. Rev. Lett. 97, 190407 (2006).

[59] D.E. Sheehy and L. Radzihovsky, Phys. Rev. Lett. 96, 060401 (2006).

[60] W.V. Liu and F. Wilczek, Phys. Rev. Lett. 90, 047002 (2003).

[61] P.F. Bedaque, H. Caldas, and G. Rupak, Phys. Rev. Lett. 91, 247002 (2003). 
[62] T. Mizushima, K. Machida, and M. Ichioka, Phys. Rev. Lett. 94, 060404 (2005).

[63] J. Carlson and S. Reddy, Phys. Rev. Lett. 95, 060401 (2005).

[64] D.T. Son and M.A. Stephanov, Phys.Rev. A 74, 013614 (2006).

[65] C.-H. Pao, Shin-Tza Wu, and S.-K. Yip, Phys. Rev. B 73, 132506 (2006).

[66] D.E. Sheehy and L. Radzihovsky, Ann. of Phys. 322, 1790 (2007).

[67] D.E. Sheehy and L. Radzihovsky, Phys. Rev. B 75, 136501 (2007).

[68] M.M. Parish, F.M. Marchetti, A. Lamacraft, and B.D. Simons, Nat. Phys. 3, 124 (2007).

[69] C. Chien, Q. Chen, Y. He, and K. Levin, Phys. Rev. Lett. 97, 090402 (2006).

[70] K.B. Gubbels, M.W.J. Romans, and H.T.C. Stoof, Phys. Rev. Lett. 97, 210402 (2006).

[71] R. Sharma and S. Reddy, Phys. Rev. A 78, 063609 (2008).

[72] C.H. Schunck, Y. Shin, A. Schirotzek, M. W. Zwierlein, and W. Ketterle, Science 316, 867 (2007).

[73] A. Schirotzek, C.-H. Wu, A. Sommer, and M.W. Zwierlein, Phys. Rev. Lett. 102, 230402 (2009).

[74] S. Nascimbène, N. Navon, K.J. Jiang, L. Tarruell, M. Teichmann, J. McKeever, F. Chevy, and C. Salomon, Phys. Rev. Lett. 103, 170402 (2009).

[75] T.-L. Ho, Phys. Rev. Lett. 92, 090402 (2004).

[76] H. Heiselberg, Phys. Rev. A 63, 043606 (2001).

[77] J. Carlson, S.-Y Chang, V. R. Pandharipande, and K.E. Schmidt, Phys. Rev. Lett. 91, 050401 (2003).

[78] G.E. Astrakharchik, J. Boronat, J. Casulleras, and S. Giorgini, Phys. Rev. Lett. 93, 200404 (2004).

[79] A. Bulgac, J. E. Drut and P. Magierski, Phys. Rev. Lett. 96, 090404 (2006).

[80] E. Burovski, N. Prokof'ev, B. Svistunov, and M. Troyer, Phys. Rev. Lett. 96160402 (2006).

[81] F. Chevy, Phys. Rev. Lett. 96, 130401 (2006).

[82] P. Nikolić and S. Sachdev, Phys. Rev. A 75, 033608 (2007)

[83] M.Y. Veillette, D.E. Sheehy, and L. Radzihovsky, Phys. Rev. A 75, 043614 (2007).

[84] L.D. Landau and E.M. Lifshitz, Quantum Mechanics: non-relativistic theory, Permagon, New York, 1977.

[85] J. Levinsen and V. Gurarie, Phys. Rev. A 73, 053607 (2006).

[86] Quantum Field Theory and Critical Phenomena, by J. Zinn-Justin (Oxford University Press, New York, 1989).

[87] M.E. Fisher, Rev. Mod. Phys. 46, 597 (1974).

[88] K.G. Wilson, Rev. Mod. Phys. 47, 773 (1975).

[89] Y. Nishida and D.T. Son, Phys. Rev. A 75, 063617 (2007).

[90] M. Veillette, E.G. Moon, A. Lamacraft, L. Radzihovsky, S. Sachdev, and D.E. Sheehy, Phys. Rev. A 78, 033614 (2008).

[91] R. Haussmann, W. Rantner, S. Cerrito, and W. Zwerger, Phys. Rev. A 75, 023610 (2007).

[92] K.E. Strecker, G.B. Partridge, R.G. Hulet, Phys. Rev. Lett. 91, 080406 (2003).

[93] D.E. Sheehy and L. Radzihovsky, Phys. Rev. Lett. 95, 130401 (2005).

[94] M. Marini, F. Pistolesi, and G.C. Strinati, Eur. Phys. J. B 1, 151 (1998).

[95] S. Pilati and S. Giorgini, Phys. Rev. Lett. 100, 030401 (2008).

[96] P. Fulde and R. A. Ferrell, Phys. Rev. 135, A550 (1964).

[97] A.I. Larkin and Yu.N. Ovchinnikov, Zh. Eksp. Teor. Fiz 47, 1136 (1964) [Sov. Phys. JETP 20, $762(1965)]$.

[98] M. Iskin and C.A.R. Sá de Melo, Phys. Rev. Lett. 97, 100404 (2006).

[99] A. Lamacraft and F. M. Marchetti, Phys. Rev. B 77, 014511 (2008).

[100] The $\mathrm{SF}_{\mathrm{M}}$ state (a global minimum at intermediate imbalance, deep in the BEC regime) resembles the (so-called) "Sarma" state, first discussed by Sarma [51] (who first showed that it is always unstable); nonetheless, the Sarma state has been mistakenly identified as a ground state of superconductors under an imposed imbalance in several subsequent publications.

[101] A. Bulgac and M.M. Forbes, Phys. Rev. A 75, 031605(R) (2007).

[102] L. Viverit, C.J. Pethick, and H. Smith, Phys. Rev. A 61, 053605 (2000).

[103] E. Taylor, A. Griffin, and Y. Ohashi, Phys. Rev. A 76, 023614(2007). 
[104] E.H. Graf, D.M. Lee and J.D. Reppy, Phys. Rev. Lett. 19, 417 (1967).

[105] R.I. Schermer, L. Passoll, and D.C. Rorer, Phys. Rev. 173, 277 (1968).

[106] R.B. Griffiths, Phys. Rev. Lett. 24, 715 (1970).

[107] A.Recati, C. Lobo, and S. Stringari, Phys. Rev. A 78, 023633 (2008).

[108] Y. Shin, C.H. Schunck, A. Schirotzek, and W. Ketterle, Nature 451, 689 (2008).

[109] Y. Shin, A. Schirotzek, C.H. Schunck, and W. Ketterle, Phys. Rev. Lett. 101, 070404 (2008).

[110] F. Chevy, Phys. Rev. A 74, 063628 (2006).

[111] R. Combescot, A. Recati, C. Lobo, and F. Chevy, Phys. Rev. Lett. 98, 180402 (2007).

[112] C. Lobo, A. Recati, S. Giorgini, and S. Stringari, Phys. Rev. Lett. 97, 200403 (2006).

[113] M. Punk, P.T. Dumitrescu, and W. Zwerger, Phys. Rev. A 80, 053605 (2009).

[114] C. Mora and F. Chevy, Phys. Rev. A 80, 033607 (2009).

[115] V. Oganesyan, S. Kivelson, and E. Fradkin, Phys. Rev. B 64, 195109 (2001).

[116] A. Sedrakian, J. Mur-Petit, A. Polls, and H. Müther, Phys.Rev. A 72, 013613 (2005).

[117] D.E. Sheehy, Phys. Rev. A 79, 033606 (2009).

[118] H.A. Radovan, N.A. Fortune, T.P. Murphy, S.T. Hannahs, E.C. Palm, S.W. Tozer, and D. Hall, Nature 425, 51 (2003).

[119] A. Bianchi, R. Movshovich, C. Capan, P.G. Pagliuso, and J.L. Sarrao, Phys. Rev. Lett. 91, 187004 (2003).

[120] K. Machida and H. Nakanishi, Phys. Rev. B 30, 122 (1984).

[121] H. Burkhardt and D. Rainer, Ann. Physik 3, 181 (1994).

[122] K. Yang, Phys. Rev. B 63, 140511 (2001).

[123] A. Bulgac and M.M. Forbes, Phys. Rev. Lett. 101, 215301 (2008).

[124] S. Matsuo, S. Higashitani, Y. Nagato, and K. Nagai, J. Phys. Soc. Japan 67, 280 (1998).

[125] N. Yoshida, and S.-K. Yip, Phys. Rev. A 75, 063601 (2007).

[126] L. Radzihovsky and A. Vishwanath, Phys. Rev. Lett. 103, 010404 (2009).

[127] J. A. Bowers and K. Rajagopal, Phys. Rev. D 66, 065002 (2002).

[128] L. Radzihovsky and A.T. Dorsey, Phys. Rev. Lett. 88, 216802 (2002).

[129] K. Yang, Phys. Rev. Lett. 95, 218903 (2005).

[130] J.M. Edge and N.R. Cooper, Phys. Rev. Lett. 103, 065301 (2009).

[131] M.M. Parish, S.K. Baur, E.J. Mueller and D.A. Huse, Phys. Rev. Lett. 99, 250403 (2007).

[132] P. Pieri and G.C. Strinati, Phys. Rev. Lett. 96, 150404 (2006).

[133] J. Kinnunen, L. M. Jensen, and P. Törmä, Phys. Rev. Lett. 96, 110403 (2006).

[134] W. Yi, L.-M. Duan, Phys. Rev. A 73, 031604 (2006).

[135] T.N. DeSilva and E.J. Mueller, Phys. Rev. A 73, 051602 (2006).

[136] A. Imambekov, C.J. Bolech, M. Lukin, and E. Demler, Phys. Rev. A 74, 053626 (2006).

[137] T.N. De Silva and E.J. Mueller, Phys. Rev. Lett. 97, 070402 (2006).

[138] X.-J. Liu, H. Hu and P.D. Drummond, Phys. Rev. A 75, 023614 (2007).

[139] M. Haque and H.T.C. Stoof, Phys. Rev. Lett. 98, 260406 (2007).

[140] L.M. Jensen, J. Kinnunen, and P. Törmá, Phys. Rev. A 76, 033620 (2007)

[141] T. Mizushima, M. Ichioka, and K. Machida J. Phys. Soc. Japan 76, 104006 (2007).

[142] J. Tempere, S.N. Klimin, and J.T. Devreese, Phys. Rev. A 78, 023626 (2008).

[143] S.K. Baur, S. Basu, T.N. De Silva, and E.J. Mueller, Phys. Rev. A 79, 063628 (2009).

[144] R.P. Feynman, in Progress in Low Temperature Physics, edited by C.J. Gorter (North-Holland, Amsterdam, 1955).

[145] A. Bulgac, M. Forbes, and A. Schwenk, Phys. Rev. Lett 97, 020402 (2006).

[146] R. Hulet, Private communication.

[147] H.-T. Shang and M.B. Salamon, Phys. Rev. B 22, 4401 (1980).

[148] W. Wu and P.W. Adams, Phys. Rev. Lett. 73, 1412 (1995).

[149] C.-C. Chien, Q. Chen, Y. He, and K. Levin, Phys. Rev. Lett. 98, 110404 (2007).

[150] M.J. Stephen, E. Abrahams, and J.P. Straley, Phys. Rev. B 12, 256 (1975).

[151] I.D. Lawrie and S. Sarbach, in Phase Transitions and Critical phenomena, ed. by C. Domb and 
J.L. Lebowitz, Vol. 9, p. 2, Academic Press (1984).

[152] G.V. Skorniakov and K.A. Ter-Martirosian, Zh. Eksp. Teor. Fiz. 31, 775 (1956) [Sov. Phys. JETP 4, 648 (1957)].

[153] D. S. Petrov, C. Salomon, G.V. Shlyapnikov, Phys. Rev. Lett 93, 090404 (2004); Phys. Rev. A 71, 012708 (2005).

[154] Y. Liao, A.S.C. Rittner, T. Paprotta, W. Li, G.B. Partridge, R.G. Hulet, S.K. Baur, and E.J. Mueller, preprint arXiv:0912.0092.

[155] G. Orso, Phys. Rev. Lett. 98, 070402 (2007).

[156] H. Hu, X.-J. Liu, and P.D. Drummond, Phys. Rev. Lett. 98, 070403 (2007).

[157] G.G. Batrouni, M.H. Huntley, V.G. Rousseau, and R.T. Scalettar, Phys. Rev. Lett. 100, 116405 (2008).

[158] A.E. Feiguin and F. Heidrich-Meisner, Phys. Rev. B 76 220508(R) (2007).

[159] M. Tezuka and M. Ueda, Phys. Rev. Lett. 100, 110403 (2008).

[160] C.H. Schunck, Y. Shin, A. Schirotzek, and W. Ketterle, Nature 454, 739 (2008).

[161] I.L. Aleiner and B.I. Altshuler, Phys. Rev. Lett. 79, 4242 (1997).

[162] Y.M. Xiong, P.W. Adams, and G. Catelani, Phys. Rev. Lett. 103, 067009 (2009).

[163] Z. Yu and G. Baym, Phys. Rev. A 73, 063601 (2006).

[164] M. Punk and W. Zwerger, Phys. Rev. Lett. 99, 170404 (2007)

[165] G. Baym, C.J. Pethick, Z. Yu, and M.W. Zwierlein, Phys. Rev. Lett, 99,190407 (2007).

[166] A. Perali, P. Pieri, and G.C. Strinati, Phys. Rev. Lett. 100, 010402 (2008).

[167] S. Basu and E.J. Mueller, Phys. Rev. Lett. 101, 060405 (2008).

[168] Y. He, C.-C. Chien, Q. Chen, and K. Levin, Phys. Rev. Lett. 102, 020402 (2009).

[169] J.T. Stewart, J.P. Gaebler, and D.S. Jin, Nature 454, 744 (2008).

[170] V.J. Emery and S.A. Kivelson, Nature 374, 434 (1995).

[171] L. Balents, M.P.A. Fisher, and C. Nayak, Phys. Rev. B 60, 1654 (1999).

[172] M. Franz, Z. Tesanovic, and O. Vafek, Phys. Rev. B 66, 054535 (2002).

[173] N.V. Prokofev, B.V. Svistunov, Phys. Rev. B 77, 020408(R) (2008); N.V. Prokofev, B.V. Svistunov, Phys. Rev. B 77, 125101 (2008).

[174] G.G. Batrouni, M.J. Wolak, F. Hebert, and V.G. Rousseau, Europhys. Lett. 86, 47006 (2009).

[175] M. Casula, D.M. Ceperley, and E.J. Mueller, Phys. Rev. A 78, 033607 (2008). 